\title{
Cultura local y regeneración urbana: un caso de estudio en Monterrey, Nuevo León
}

\section{Local culture and urban regeneration: A case study in Monterrey, Nuevo León}

\author{
Fabiola Garza-Rodríguez* \\ Elisabet Roca** \\ Míriam Villares***
}

\section{Resumen}

Desde hace varias décadas, la cultura se ha utilizado como instrumento de regeneración urbana para revitalizar las zonas degradadas. Si bien existe gran cantidad de investigaciones que estudian el fenómeno, hace falta ampliar el debate hacia las etapas de diagnóstico y planeación. Este artículo busca responder a ese vacío mediante el estudio de la técnica del mapeo cultural y de su aplicación en un caso de estudio: el centro de Monterrey, México, para identificar zonas potenciales de aglomeración de cultura. Estas zonas, conocidas como clústeres culturales, pudieran estar generando procesos de regeneración urbana que contribuyen a la revitalización del lugar. Se utiliza un método mixto de análisis que conjunta datos cuantitativos con métodos cualitativos. Los resultados muestran la existencia de dos clústeres culturales en el centro, conformados por distintos actores, diferenciados por variados tejidos urbanos, que ayudan a potenciar la regeneración del centro de Monterrey.

Palabras clave: sostenibilidad sociocultural; regeneración urbana; mapeo cultural.

* Universidad Autónoma de Nuevo León, Facultad de Arquitectura. Dirección: Pedro de Alba s/n, Ciudad Universitaria, 66455, San Nicolás de los Garza, Nuevo León, México. Correo: fgarzar@uanl.edu.mx ORCID: http://orcid.org/0000-0001-5971-0478

** Universitat Politècnica de Catalunya. Dirección: Jordi Girona, 1-3 08034 Barcelona, España. Correo: elisabet.roca@upc.edu ORCID: elisabet.roca@upc.edu

*** Universitat Politècnica de Catalunya. Correo: miriam.villares@upc.edu ORCID: https://orcid.org/0000-0001-6910-931X 


\begin{abstract}
For several decades, culture has been used as an instrument of urban regeneration to revitalize degraded areas. Although there is a great deal of research studying the phenomenon, it is necessary to extend the debate to the diagnostic and planning stages. This article seeks to fill this gap by studying the technique of cultural mapping and its application in a case study: downtown Monterrey in Mexico, to identify potential areas of culture agglomeration. These areas, known as cultural clusters, could be creating urban regeneration processes that contribute to the revitalization of the place. A mixed method of analysis is used that combines quantitative data with qualitative methods. The results show the existence of two cultural clusters in the center, comprising different actors, distinguished by various urban fabrics, which help promote the regeneration of downtown Monterrey.
\end{abstract}

Keywords: sociocultural sustainability; urban regeneration; cultural mapping.

\title{
Introducción
}

El estudio de la sostenibilidad va incorporando cada vez más perspectivas e incluye más ámbitos de análisis, justificando su importancia en el discurso político, social, ambiental, económico, urbano y cultural. De ahí que la cultura haya pasado a considerarse el cuarto pilar del desarrollo sostenible (Hawkes, 2001). En la sostenibilidad urbana, la cultura se ha vuelto una herramienta para mejorar las dinámicas sociales en el entorno urbano (Colantonio, Dixon, Ganser, Carpenter y Ngombe, 2009; Axelsson, Angelstam, Degerman, Teitelbaum, Andersson, Elbakidze y Drotz, 2013; Duxbury, 2014; Soini y Birkeland, 2014). Autores como Hawkes (2001), Ghilardi, (2003), Montgomery (2003), entre otros, consideran que la cultura contribuye a la planeación urbana y a mejorar la forma en que la sociedad habita la ciudad.

Pero un concepto tan profundo debe precisarse. Para definirlo, tomamos de referencia el libro de Terry Eagleton (2001), La idea de la cultura, donde el autor explora distintos significados y ramificaciones etimológicas de la palabra, argumentando que muchas de sus definiciones en ocasiones resultan contradictorias, terminando por fragmentar un concepto que siempre ha sido universal (Eagleton, 2001). Esta fragmentación del concepto de cultura (siendo más evidente en la época posmoderna) hace que se aligere el sentido de identidad, perdiendo la raíz del significado de la cultura humana como parte esencial de la sociedad. De ahí que el autor la defina como "el conjunto de valores, costumbres, creencias y prácticas que constituyen la forma de vida de un grupo específico" (Eagleton, 2001, p. 58). Dentro de 
ese mismo eje se ubica la definición de Hawkes (2001), quien ve a la cultura como:

[...] la forma de vida de un conjunto particular de seres humanos [...] producción y transmisión de identidades, significados, conocimiento, creencias, valores, aspiraciones, memorias, propósitos, actitudes y entendimiento [Hawkes, 2001, p. 3].

Por lo tanto, entendemos la cultura como aquello que reconoce valores en el entorno físico y social, permitiendo identificar el valor cultural de cada lugar, llámese: patrimonio, geografía, historia, identidad, arquitectura, estilo de vida, sentido de pertenencia, tradiciones, costumbres locales, ritos, creencias, fotografías, memorias colectivas o relatos.

Al trasladar este par de definiciones hacia la perspectiva del desarrollo sostenible es cuando entendemos que la cultura es fundamental para la planeación y la creación de políticas públicas urbanas, cuyo propósito es lograr la transición hacia ciudades y comunidades sostenibles (Hawkes, 2001; Duxbury, Garrett-Petts y MacLennan, 2015). Sin embargo, uno de los obstáculos más comunes al hablar de cultura es la marcada diferencia entre alta cultura y cultura de masas. La primera responde a la producción cultural y artística, siendo exclusiva de ciertas élites, mientras que la segunda toma elementos locales que conforman las prácticas de una comunidad, la cultura popular o los valores que forman e identifican a una sociedad (Lipovetsky, 1994; Hawkes, 2001). En la época actual, la alta cultura ha pasado a la historia, ya no busca ser exclusiva de ciertas élites, ni generar productos culturales y artísticos para un público distintivo, sino que busca ser democrática. Se propone alejarse del concepto tradicional de cultura para pasar a entenderla como aquella que impregna todas las actividades de la vida cotidiana, que no es exclusiva de ciertas actividades ni de ciertas élites. El modelo al que se orienta el discurso cultural actual busca la democratización de la cultura (Vargas Llosa, 2012), no su privatización. Por lo tanto, el reto de la sostenibilidad y de la cultura como parte esencial del desarrollo consiste en ser eje y motor de los procesos de planeación, transformación y regeneración urbana, en donde se involucre no sólo la producción artística e infraestructura cultural, sino también las percepciones, la identidad y el sentido de pertenencia, valores culturales que definen a la sociedad y que influyen en su forma de habitar la ciudad.

Ante la creciente demanda urbana, las ciudades deben fomentar el desarrollo humano, así como brindar igualdad de oportunidades para sus habitantes, por lo que la ciudad debe apostar por la equidad de los espacios 
de convivencia que fomenten y promuevan la libertad, la paz, la recreación, los espacios verdes y los culturales, que generen bienestar y felicidad; tales cuestiones son cada vez más valoradas en la búsqueda de una buena calidad de vida (Papachristou y Rosas-Casals, 2018).

Al hacer una aproximación al contexto latinoamericano, se aprecia que las áreas más deprimidas de las ciudades son los centros históricos o los centros de ciudad. Hablando genéricamente, los centros cuentan con alta riqueza arquitectónica, urbana y cultural por el simple hecho de contener los trazados más antiguos de la ciudad. Sin embargo, también son lugares que sufren procesos de abandono y pérdida de población. La expansión territorial, la migración de la población del centro hacia la periferia, el acelerado crecimiento demográfico y el discurso mal enfocado de modernidad (Balbó, Jordán y Simioni, 2003; Bazant, 2012; Prieto González, 2010) son sólo algunos factores que han contribuido en su declive. Las propuestas para rehabilitar los centros resultan en dinámicas de planificación que involucran regeneraciones orientadas a la reactivación de los sectores marginados mediante estrategias de transformación urbana desde las asociaciones entre los sectores público y privado. De manera general, los procesos de regeneración se orientan al mercado capitalista, tendiendo a la gentrificación más que a lograr transformaciones de raíz, debido a las nuevas condiciones de renta y al uso que deliberadamente o no, se imponen.

Éste es un fenómeno recurrente en los centros, y así sucede en Monterrey: se pierde identidad social y patrimonio histórico- arquitectónicocultural (Balbo, Jordán y Simioni, 2003) ante la apropiación de espacios por parte de actores públicos y privados para su capitalización (Becerril-Padua, 2000; Casgrain y Janoschka, 2013). Los centros suelen verse como zonas desprotegidas y desarraigadas de cultura. Esto sucede particularmente en Monterrey, donde, además, la población local manifiesta un "complejo de inferioridad urbana" -si se le puede llamar así- al comparar el centro de la ciudad con centros históricos del centro y sur de México, dotados de mayor patrimonio cultural. Este pensamiento infructuoso se ha impregnado en la memoria colectiva del regiomontano, haciéndole creer que Monterrey no tiene cultura. Sin embargo, esta visión, por demás errada, surge del desconocimiento por parte de la población local de que la cultura en Monterrey se ha creado y entendido de forma alterna. Debido a su carácter manufacturero, la cultura ha estado ligada al espíritu empresarial e industrial; de ahí que las prácticas culturales en Monterrey a lo largo del siglo XX surjan a partir de los corporativos más importantes de la ciudad, como grupo Alfa o Cervecería Cuauhtémoc Moctezuma (Ramírez, 2009, p. 10). 
En los últimos sesenta años, Monterrey se ha desarrollado a un ritmo acelerado. Su población se dobló en la década de los cincuenta y su centro comenzó a perder población de manera gradual debido a la migración de población a la periferia. El centro de Monterrey se conforma de muchos centros; más allá de sus límites establecidos, ${ }^{1}$ es un área compuesta por diversos barrios de especial importancia histórica y arquitectónica, siendo sede de funciones administrativas, culturales y turísticas del área metropolitana.

Esta investigación discute el proceso de regeneración urbana llevado a cabo en el centro de Monterrey y, utilizando la técnica del mapeo cultural, identifica la existencia de clústeres culturales en el centro que pudieran indicarnos distintos o nuevos modelos de regeneración urbana. El mapeo cultural se complementa con la aplicación de entrevistas en profundidad, analizadas a partir de narrativas urbanísticas, para entender desde diversas perspectivas de análisis la situación sociourbana del centro. En ese sentido, los objetivos específicos del estudio son los siguientes:

- Analizar el proceso de regeneración urbana implementado en el centro de Monterrey en los últimos veinte años.

- Mapear y analizar la distribución geográfica de los recursos culturales para identificar su concentración, lo que puede indicarnos la existencia de un clúster cultural en el territorio.

- Crear una radiografía cultural del centro de Monterrey que nos permita reconocer sus principales amenazas y propuestas de mejora sociourbanística.

La investigación contribuye al estudio de la sostenibilidad cultural en el urbanismo y en las formas en que la cultura se manifiesta en lo urbano. Específicamente, el trabajo busca visibilizar la técnica de mapeo cultural en el contexto latinoamericano como herramienta de diagnosis y análisis; hasta ahora, no se tiene conocimiento de que se haya analizado desde esta perspectiva en ciudades del norte de México.

${ }^{1}$ El borde del centro de Monterrey se conoce como "primer cuadro", denominación que abarca el territorio entre la avenida Colón (norte), Constitución (sur), Félix U. Gómez (oriente) y Venustiano Carranza (poniente). Cuando se hace referencia al centro histórico, se alude al polígono Barrio Antiguo. 


\section{El discurso cultural en la regeneración urbana y el mapeo cultural como técnica de análisis urbano}

Como alternativa para transformar el entorno urbano habitado, organizaciones internacionales, gobiernos locales e investigadores han apostado por incluir la cultura como herramienta de regeneración, planeación y mejora urbana (Montgomery, 2003). Se ha demostrado que, en lo urbano, la cultura produce beneficios económicos (Markusen y Schrock, 2006), así como innovación y conocimiento sobre el lugar donde coexiste. Sin embargo, también es productora de efectos negativos vinculados al progreso, siendo común que produzca fenómenos asociados a la gentrificación y la segregación (Zukin, 1982; Peck, 2005).

La regeneración urbana es un proceso sistémico que parte de una estrategia política que, mediante regulaciones y planeación, promueve inversiones con la finalidad de transformar un lugar con deterioro físico, social, ambiental, económico y cultural (Evans, 2005; Roberts, Sykes y Granger, 2016). Si bien en un inicio el interés era meramente económico (Jones y Evans, 2008; Pastak y Kährik, 2016), hoy en día sus objetivos van más allá y buscan promover, a través de nuevas estrategias y enfoques -siendo la cultura uno de ellos-, ciudades inclusivas y sostenibles (García, 2004; Tavano Blessi, Tremlay, Sandri y Pilati, 2012; Duque Franco, 2015). El reto urbano actual es crear ciudades compactas, saludables, equitativas, eficientes, creativas y cohesionadas (Evans, 2005; Florida, 2005), factores cualitativos de bienestar que, como se ha demostrado, influyen de manera directa en la resiliencia urbana y en la calidad de vida de sus habitantes (Heath, Rabinovich y Barreto, 2017). Ciudades europeas, australianas, canadienses e inclusive asiáticas, ya han apostado por modelos de regeneración urbana basados en la cultura (García, 2004; Jones y Evans, 2008; Kana, 2012; Jung, Lee, Yap e Ineson, 2015). En el contexto latinoamericano han sido estudiados y analizados casos de ciudades que han apostado por este tipo de regeneración (véase Yúdice, 2008; Kanai y Ortega-Alcázar, 2009; Becker y Müller, 2013; Duque Franco, 2015; Rius-Ulldemolins y Posso, 2016), siendo las ciudades colombianas de Medellín y Bogotá los ejemplos y paradigmas que apuestan y materializan la cultura como modelo político de intervención y regeneración urbana.

El potencial para mejorar la planeación de las ciudades y la forma en que la sociedad habita en ellas es una de las inquietudes del estudio de lo urbano desde la perspectiva cultural. De ahí que la sostenibilidad cultural sea vista como el proceso y las características que construyen, mantienen, refuerzan e identifican los valores esenciales de una sociedad. De mane- 
ra sistémica, identifica, categoriza y recoge información sobre los recursos y valores culturales que identifican a una comunidad (Evans y Foord, 2008; Stern y Seifeit, 2010; Duxbury, Garret-Petts, MacLennan, 2015; Freitas, 2016; Cauchi-Santoro, 2016), con la finalidad de visibilizarlos y tomarlos como punto de partida para proponer estrategias de revalorización urbana.

El mapeo cultural desde la visión del desarrollo sostenible es el equilibrio entre la preservación de los bienes culturales y el cumplimiento de sus propios objetivos (Duxbury, Garrett-Petts, MacLennan, 2015; Freitas, 2016). Es una técnica para identificar aglomeraciones de recursos y actividades culturales que surgen de forma orgánica o natural (Stern y Seifert, 2010), de manera deliberada o partiendo de una política del gobierno local (Montgomery, 2003; Park, 2016). La información cultural existe en todas las comunidades, pero comúnmente se encuentra fragmentada. Los bienes culturales se componen tanto de recursos tangibles como intangibles. Los recursos tangibles son: espacios físicos, organizaciones culturales, arte público, industrias culturales, patrimonio natural y cultural, arquitectura, personas, artefactos (Duxbury, Garret-Petts y MacLennan, 2015), cafés culturales, galerías, edificios patrimoniales, museos, escuelas (Cauchi-Santoro, 2016; Jeannotte, 2016). Los recursos intangibles se relacionan con valores, normas, creencias, narrativas de la comunidad, lenguaje, historias y memorias, rituales, tradiciones, identidades y sentido de pertenencia (Duxbury, Garret-Petts y MacLennan, 2015). Desafortunadamente, los recursos intangibles son menos explorados por la investigación científica debido a que tienden a la subjetividad. Dentro de esa línea, Jeannotte (2016) y Cauchi-Santoro (2016) han realizado mapeos culturales enfocándose en visibilizar valores intangibles en centros de ciudades canadienses, pero hacen falta más contribuciones a esta aproximación de análisis sociocultural del territorio, y también se requieren desarrollos metodológicos más específicos.

Los mapeos culturales, así como las regeneraciones urbanas, pueden realizarse desde procesos top-down o bottom-up (Montgomery, 2003; Freitas, 2016; Park, 2016). El enfoque top-down se ejecuta por expertos que utilizan técnicas cuantitativas de análisis para proponer estrategias y modelos de intervención. El proceso bottom-up es más horizontal y participativo; está basado en técnicas cualitativas en donde actores locales (residentes, organizaciones civiles) definen el proceso y las estrategias a seguir partiendo de necesidades locales (Freitas, 2016). El fin último del mapeo cultural es la creación de políticas culturales, asignación de fondos, creación o mejora de infraestructura, planeación pública urbana (Freitas, 2016), implementación de turismo cultural, rutas y paseos cul- 
turales, revalorización de un territorio (Cauchi-Santoro, 2016), recuperación de patrimonio cultural, histórico y arquitectónico; y, además, ayuda a reconocer la existencia de concentraciones de cultura en un territorio, coadyuvando a la identificación de zonas donde suceden dinámicas culturales específicas: los llamados clústeres culturales.

El aporte de la cultura a la ciudad se manifiesta a través de estrategias enfocadas en la creatividad, en medios de expresión artística, en el desarrollo de la identidad colectiva (Zukin, 1987; Harvey, 1989; Bianchini y Parkinson, 1994; Landry, 2005), en la creación de macroeventos y proyectos emblemáticos (García, 2004; Tallon, 2013; Jung, Lee, Yap e Ineson, 2015), en la implementación de políticas públicas culturales (Bianchini y Parkinson, 1994; Landry y Bianchini, 1995) y de gobernanza urbana (Degen y García, 2012). Tales estrategias culturales, más allá de buscar el crecimiento económico, generan métodos alternativos de desarrollo urbano y social, contribuyendo al discurso de sostenibilidad urbana, no sólo para perfeccionar la forma y funcionamiento de la urbe, sino para mejorar la experiencia de vivir en ella (Landry y Bianchini, 1995).

\section{Metodología}

La metodología de esta investigación parte de la revisión de trabajos académicos, documentos históricos y planes urbanísticos locales, complementada con entrevistas en profundidad realizadas a actores clave, junto con la observación directa sobre el lugar para construir los antecedentes y definir la situación actual del centro. Para identificar y caracterizar la presencia de clústeres culturales en el centro de Monterrey, se utiliza un método mixto que combina técnicas cuantitativas, cualitativas y cartográficas. La investigación parte de un análisis del contexto urbano, su historia y evolución urbanística, así como del análisis del planeamiento y las políticas urbanas implementadas (Garza-Rodríguez, 2015). Se realizó un levantamiento cartográfico de los recursos culturales tangibles a escala de área geoestadística básica (AGEB), unidad territorial utilizada por el Instituto Nacional de Estadística y Geografía (INEGI).

Los recursos culturales se cartografiaron a partir de la categorización propuesta en el Cuadro 1. Se realizó mediante un análisis descriptivo que involucra información obtenida de la Guía de patrimonio arquitectónico de Nuevo León (Mancillas Hinojosa, 2009), de libros (Prieto González, 2014; Casas García, 2015) y del portal web del Sistema de Información 
Cultural, ${ }^{2}$ el cual es una base de datos del sector público que concentra los recursos culturales existentes en el país, dividida por estados. A partir de las consultas a las referencias descritas, junto con la observación directa del sitio, se cartografió, mediante sistemas de información geográfica (SIG), la mayor parte de los recursos culturales presentes en el centro de Monterrey. En cuanto a los espacios culturales independientes (ECI), se realizó una investigación exploratoria para identificarlos, a través de las redes sociales, construyendo una base de datos para después representarlas en el SIG.

\section{Cuadro 1}

Categorización de recursos culturales tangibles

\begin{tabular}{ll}
\hline \multicolumn{1}{c}{ Categorías } & \multicolumn{1}{c}{ Recursos culturales tangibles } \\
\hline Equipamientos culturales & Museos, teatros, bibliotecas, recintos para eventos \\
\hline Centros educativos & Escuelas secundarias, preparatorias y universidades \\
\hline Elementos patrimoniales- & Centros espirituales / religiosos \\
simbólicos & y monumentos históricos \\
\hline Espacio público & Plazas públicas y áreas verdes \\
& Espacios culturales, galerías de arte, editoriales, \\
& librerías, cafés culturales, comercio local, antigüedades \\
\hline
\end{tabular}

Fuente: Elaboración propia.

Una vez introducida cada variable en el SIG, se calcularon buffers de 600 metros por cada recurso cultural. Este valor $(600 \mathrm{~m})$ es un dato que surge de la consulta bibliográfica (Rueda, Cuchí, De Cáceres y Brau, 2011), pues es la distancia que en términos peatonales representa 10 minutos andando. El cálculo de los buffers ayuda a reconocer áreas con mayor concentración y proximidad de recursos culturales, lo que nos permite identificar de manera preliminar la posible existencia del clúster cultural en el centro, al menos desde la georreferencia.

Para el análisis complementario del centro a partir de narrativas, se realizaron 14 entrevistas en profundidad a informantes clave, los cuales tienen un amplio conocimiento sobre la situación sociourbana del centro de Monterrey. Fueron elegidos debido a que basan su investigación, activismo o trabajo profesional en estudiar e intervenir el tejido urbano del centro. Los informantes son cuatro catedráticos de la Facultad de Arquitectura de la Universidad Autónoma de Nuevo León (UANL), tres activistas locales

\footnotetext{
${ }^{2}$ http://sic.gob.mx/index.php?table=auditorio\&estado_id $=19$
} 
y representantes de colectivos, un promotor cultural, un periodista cultural, cuatro arquitectos de la iniciativa privada, y el exdirector del Instituto de Planeación Urbana y Municipal de Monterrey (ImplancMty). Un rasgo en común entre los informantes es que la mayor parte de ellos habita en el centro, lo que brinda un valor agregado a las entrevistas, ya que, además, son residentes permanentes del lugar.

Las preguntas formuladas permitieron construir narrativas sobre lo que sucede en el centro de Monterrey. Las entrevistas comenzaron identificando las problemáticas urbanas, sus orígenes y las estrategias para su regeneración. Se preguntó también sobre las pequeñas y grandes intervenciones urbanas realizadas y si se han llevado a cabo a partir de procesos de participación ciudadana o no. El aspecto cultural se enfocó en conocer el potencial del centro en relación con su espacio público y su patrimonio cultural y arquitectónico, preguntando también si existe una buena cobertura de equipamientos culturales. Las entrevistas finalizaron con propuestas de soluciones de mejora para las cuestiones encontradas. Todas se realizaron de forma presencial y de manera individual durante diciembre de 2015 y enero de 2016 en Monterrey, México; fueron audiograbadas y transcritas para su posterior análisis. Para el análisis de contenido, se optó por clasificar categorías y conceptos clave identificados a partir de las narrativas reiterativas. Cuando una respuesta era similar a otra, se identificó con una frase que sintetiza la narrativa expuesta, y se etiquetó de mayor a menor mención. Esta categorización de datos no significa que una narrativa sea más importante que otra, sólo refleja la reincidencia de un mismo discurso, de una misma manera de pensar sobre una pregunta concreta.

\section{Aproximación al caso de estudio: el centro de Monterrey}

Localizada al noreste de México, la ciudad de Monterrey es la capital del estado de Nuevo León. Su área metropolitana es la tercera más poblada del país, concentrando una población de casi cuatro millones y medio de habitantes en 2017. En términos geoeconómicos, su ubicación es privilegiada, pues se encuentra a menos de $250 \mathrm{~km}$ de la frontera con Estados Unidos, lo que ha impulsado el carácter dinámico e industrial de la región. El desarrollo económico del área metropolitana se basa principalmente en el sector industrial, manufacturero y de servicios; sin embargo, desde hace más de 15 años el gobierno estatal ha apostado por diversificar el desarrollo hacia los sectores de la innovación y el conocimiento (Moreno y Contreras, 2010), con la finalidad de generar competitividad urbana y posicionar a Monterrey 
como ciudad global. El salto por diversificar el desarrollo económico se produce para convertirla en ciudad del conocimiento y orientar su desarrollo hacia el sector creativo y cultural (Villarreal González, Gasca Sánchez y Flores Segovia, 2016).

En contraparte al dinamismo global que proyecta la ciudad y su área metropolitana, ésta presenta una serie de elementos negativos que afectan su imagen urbana y, más importante aún, la calidad de vida de sus habitantes. Por ejemplo, en 2018, la Organización Mundial de la Salud (OMS) declaró que el área metropolitana de Monterrey es la más contaminada de México. ${ }^{3}$ De hecho, en 2017 sólo 27\% de los 365 días del año hubo buena calidad del aire (Martínez, 2018), dato que es, por demás, preocupante. A la alta contaminación del aire se suman la deficiente red de transporte público, la falta de regulaciones y de políticas públicas para la planeación y el crecimiento de la ciudad y los altos índices de corrupción, por mencionar sólo algunos problemas. Además, el área metropolitana de Monterrey no se desarrolla a la par que su centro, como Prieto González (2013) argumenta, haciendo alusión a que su crecimiento y desarrollo "se dan de manera simultánea, pero mientras la primera se muestra al mundo con orgullo, la segunda se silencia, se oculta, a pesar de su abrumador dominio y existencia previa" (p. 18). El centro de Monterrey, como otros tantos centros de ciudades latinoamericanas, sufre fenómenos de despoblación, abandono y degradación a favor del crecimiento de las periferias, así como un marcado problema de segregación socioespacial (para revisar algunos ejemplos, véase Rojas, 2004; Aparicio, Ortega y Sandoval, 2011), que contrasta con la imagen de Monterrey que el sector público y el privado buscan mostrar al mundo. Desde la década de los ochenta, es en el centro donde se ha apostado por realizar regeneraciones urbanas que surgen desde políticas administrativas, municipales y privadas, cuyo objetivo principal ha sido mejorar la imagen urbana para atraer turismo y seguir con la diversificación económica ya comentada (para ampliar la información al respecto, véase Prieto González, 2013; 2014; 2015).

El centro de Monterrey, tomando como límite el cerro del Obispado al oeste y el Parque Fundidora al este, cuenta con una superficie aproximada de 1100 hectáreas, y está conformado por 765 manzanas (Garza-Rodríguez, 2014). Según cálculos propios, tomando los datos más recientes del Censo General de Población y Vivienda de 2010 del INEGI, se estima que cuenta aproximadamente con 30000 habitantes. Territorialmente, el $70 \%$ de su área está ocupada, mientras que el $30 \%$ restante se encuentra en abandono.

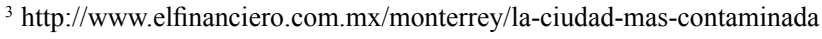


Los datos del INEGI también arrojan que la mayor parte de sus manzanas tienen entre 1 y 40 habitantes, mientras que 162 de ellas prácticamente no cuentan con ellos.

\section{Mapa 1}

Límites del centro de Monterrey establecidos para esta investigación

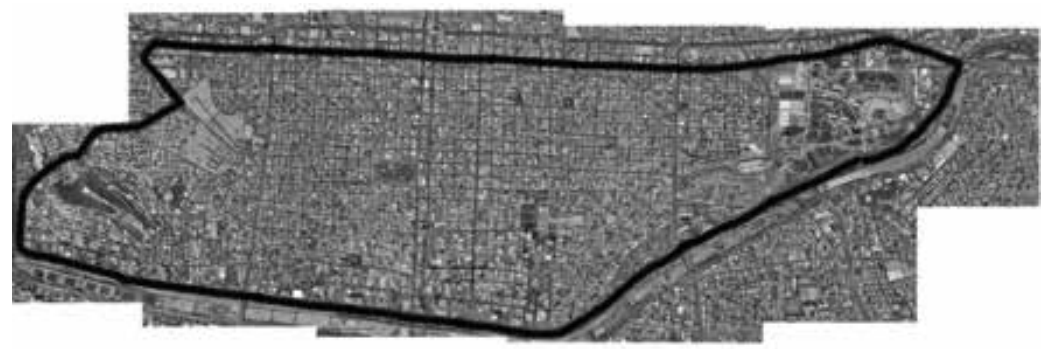

Fuente: Elaboración propia.

\section{Fotografía 1}

Inmuebles en el centro en estado de abandono y deterioro

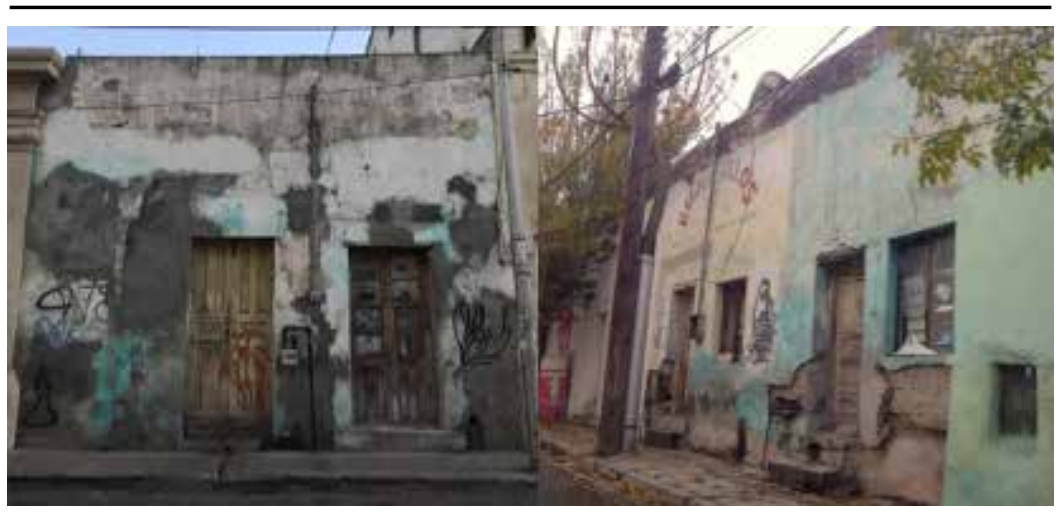

Fuente: Fotografía de Fabiola Garza-Rodríguez. 


\section{Breve repaso del proceso de regeneración y de las políticas de transformación urbano-culturales}

La cultura en Monterrey está fuertemente ligada a la industria empresarial (Ramírez, 2009; Prieto González, 2015). Durante todo el siglo XX, el desarrollo de la ciudad se ha caracterizado por fundir capital y cultura (Ramírez, 2009), peculiaridad visible a partir de la creación de la Macroplaza, el Parque Fundidora, el eje Macroplaza-Parque Fundidora mediante el Paseo Santa Lucía, el Fórum Universal de las Culturas, la rehabilitación del Barrio Antiguo, por mencionar algunos ejemplos. Tales obras corresponden a estrategias culturales orientadas a planes de renovación urbana mediante la creación de proyectos emblemáticos, equipamientos culturales o megaeventos (Bianchini y Parkinson, 1994; Duque Franco, 2015), cuyo objetivo es revitalizar un área urbana mediante la presencia de un ejemplar arquitectónico de marca, o siendo sede de algún evento internacional, como por ejemplo, el caso del Guggenheim en Bilbao (Lange-Valdés, 2018) o el Fórum Universal de las Culturas.

Dichas estrategias culturales de regeneración, impulsadas por asociaciones entre el sector público y el privado, catalogan a Monterrey como "ciudad del espectáculo", argumento que ya ha discutido Prieto González (2013, p. 18), haciendo referencia a los esfuerzos por atraer turismo e inversiones a la ciudad. Sin embargo, el sector privado ha jugado un rol fundamental como constructor y gestor de la cultura en Monterrey. Tanto, que ésta no puede concebirse sin incluir a los grandes corporativos de la ciudad: "los empresarios utilizaron la cultura como recurso para fines como identidad empresarial y nacional, manejo político y atracción de capital, creando un contexto atípico" (Ramírez, 2009, p. 2), donde la cultura se relaciona con capital, con negocio. Prueba de ello son las intervenciones realizadas, en donde más allá de impulsar el desarrollo cultural, se sobreponen los beneficios económicos. Las problemáticas vividas hoy en día en torno a las políticas culturales en Monterrey surgen porque no se han podido separar las alianzas e intereses entre el sector público y el privado (Ramírez, 2009). A continuación, presentamos un par de ejemplos.

Unos cuantos años después de la inauguración de la Macroplaza, el entonces gobernador del estado, Sócrates Rizzo, propuso la creación de la Ley de Patrimonio Cultural del Estado de Nuevo León (1991), con la finalidad de salvaguardar el patrimonio histórico, artístico y cultural del estado, y en 1993 se aprobó el decreto que declara al Barrio Antiguo como "zona protegida con carácter de centro histórico" (Reglamento del Barrio Antiguo de Monterrey, 1993), polígono compuesto por 16 manzanas (Mendoza Lemus, 
2018). Esta ley surgió también para proteger el patrimonio cultural del estado de Nuevo León. A poco más de un cuarto de siglo de su aprobación, la Ley de Patrimonio Cultural del Estado de Nuevo León no ha sido respetada ni seguida por las autoridades ni por el sector privado, ya que se siguen destruyendo casonas antiguas catalogadas como patrimonio arquitectónico (Mendoza Lemus, 2017), perdiéndose memoria e identidad, alterando además el contexto del lugar.

En cuanto a las políticas culturales, sólo un organismo público las maneja en el estado: el Consejo para la Cultura y las Artes de Nuevo León (Conarte). Creado en 1995, surgió "para modernizar y transparentar el manejo de la cultura gubernamental" (Ramírez, 2009, p. 75), en la que se busca promover las artes y la cultura del estado, así como salvaguardarlas. Su creación fue innovadora: se propuso que fuera un organismo público descentralizado a cargo de la sociedad civil. Dentro del Programa Especial de Cultura del Gobierno del Estado de Nuevo León (2017, p. 9) se define la labor del Conarte:

[...] contribuir al desarrollo humano y social [...] brindarles acceso a los bienes y servicios culturales, divulgar expresiones artísticas, impulsar a los creadores, sensibilizar las artes y reconocer el valor de la cultura popular y el patrimonio tangible e intangible, para propiciar la construcción de identidad, ciudadanía y comunidad, con respeto a la diversidad cultural existente.

Sin embargo, hoy en día, la cultura se maneja de manera centralizada, lo que no es lo ideal porque produce problemas burocráticos que no deberían existir ante un elemento tan etéreo como la cultura. Como bien apunta Zaid (2013), "el centralismo ahoga a nuestra vida cultural tanto como a nuestra imperfecta democracia política" (p. 37). La centralización es un factor que Conarte reconoce como una desventaja. De hecho, en mayo de 2017 se firmó un acuerdo en conjunto con el Consejo Nuevo León para la Planeación Estratégica para realizar un diagnóstico sobre las políticas culturales del estado, medir su impacto económico e identificar las actividades y manifestaciones culturales que impulsen el desarrollo cultural (Hora Cero, 2017). El resultado se presentó en noviembre de 2017 por medio de los documentos Evaluación diagnóstica de las políticas culturales del Estado de Nuevo León (1995 a 2017), ${ }^{4}$ y Análisis del sector cultural del Estado de Nuevo León, ${ }^{5}$ los cuales muestran la situación en la que se encuentra

\footnotetext{
${ }^{4}$ https://conl-mx.s3.amazonaws.com/documents/document_files/000/000/039/original/ publi_02_v1.pdf? 1512582688

${ }_{5}^{5}$ https://conl-mx.s3.amazonaws.com/documents/document_files/000/000/042/original/ publi_03_v1.pdf? 1513360723
} 
la entidad respecto a sus políticas culturales, identifican fortalezas y hacen propuestas de mejora para impulsar el desarrollo cultural en la ciudad. Esto no se había hecho desde la creación de Conarte.

Es necesario impulsar el desarrollo cultural de la ciudad a través de políticas culturales integrales y descentralizadas. Como hemos visto, la política de mejora o revitalización del centro de Monterrey ha estado marcada por un incremento de proyectos de regeneración urbana que surgen desde el sector público y el privado; desde la creación de proyectos emblemáticos y equipamientos culturales, de embellecimiento y mejora estética y urbana para promover el turismo, hasta proyectos de oferta inmobiliaria de usos mixtos por parte del sector privado. Sin embargo, a la par, han surgido proyectos impulsados por iniciativas ciudadanas cuyo fin también es regenerar el centro. La diferencia entre las intervenciones urbanísticas realizadas por el sector público-privado es que, en este caso, son los residentes locales quienes promueven, gestionan y financian las intervenciones. El Cuadro 2 sintetiza los proyectos desarrollados en el centro de Monterrey en las últimas dos décadas. Los clasificamos en categorías de acuerdo con su tipología: a) renovación urbana, $b$ ) rehabilitaciones arquitectónicas, $c$ ) nueva construcción $\mathrm{y}, d$ ) participación comunitaria.

\section{a) Proyectos de renovación urbana}

Los proyectos de renovación urbana son impulsados por el sector público-privado. La mayor parte de éstos han quedado en el papel. Se mencionan a continuación los que se encuentran dentro de esta tipología.

La peatonalización de la calle Morelos en Barrio Antiguo, realizada en 2014 con una inversión de 20 millones de pesos, con la que se buscaba "impulsar la vocación cultural e histórica" del barrio (Alanís, 2014).

El proyecto Emporio fue un programa organizado por la Asociación Mexicana de Profesionales Inmobiliarios (AMPI) de Monterrey y estuvo apoyado por el Colegio de Arquitectos de Nuevo León (El Horizonte, 2015). Buscaba impulsar proyectos inmobiliarios a través de inversiones derivadas de asociaciones entre el sector público municipal y el privado, cuyas especificaciones consistían en regenerar terrenos baldíos, inmuebles abandonados y subutilizados localizados en el centro de la ciudad. A pesar de que el programa contaba con buenas intenciones y seguía un modelo de gestión similar a otro en la Ciudad de México, no prosperó y el programa no continuó. 


\section{Cuadro 2}

Clasificación de proyectos de regeneración en el centro

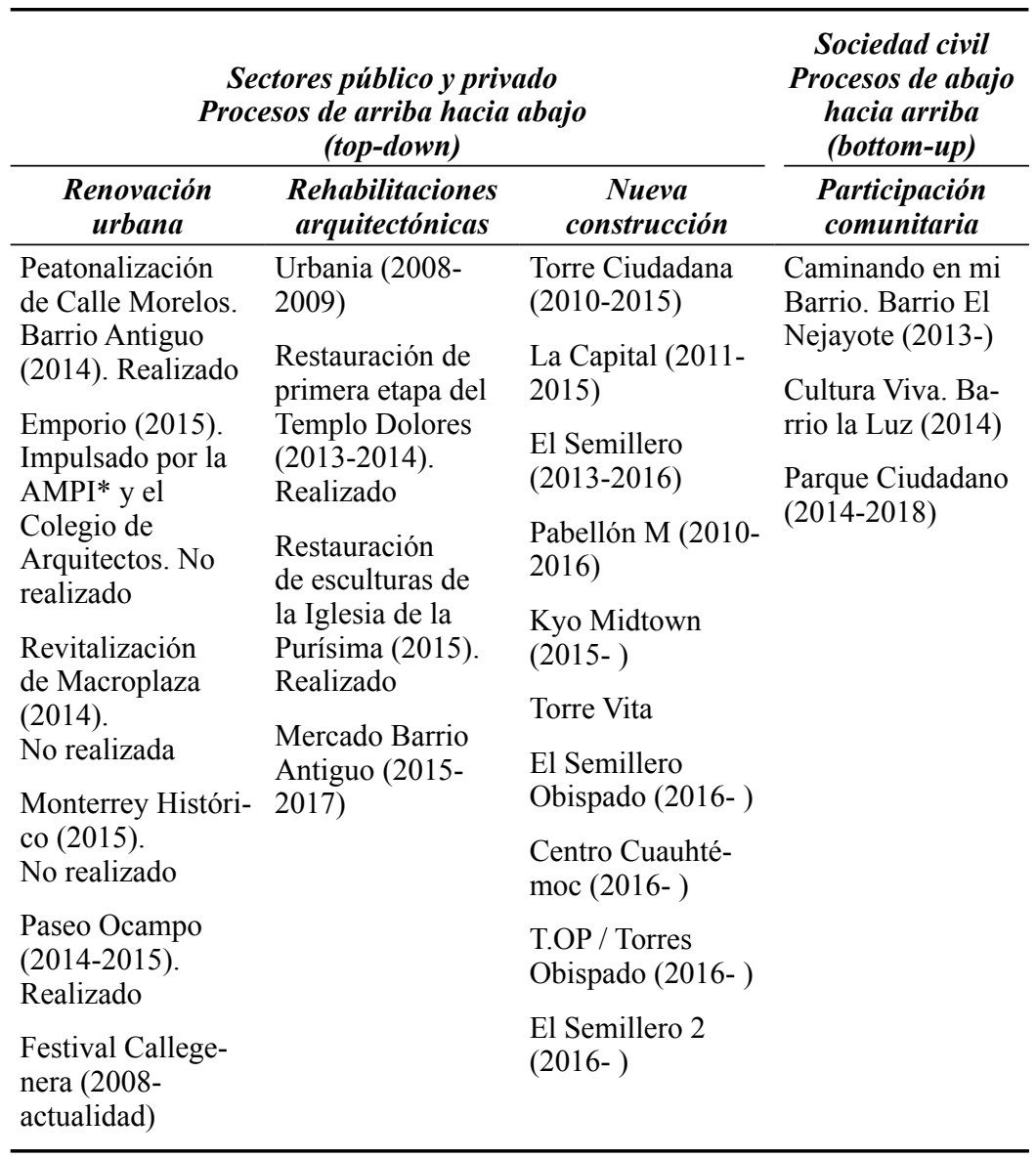

* Asociación Mexicana de Profesionales Inmobiliarios.

Fuente: Elaboración propia.

Otro proyecto es el de la revitalización de la Macroplaza, el cual se sometió a concurso público; sin embargo, estuvo cargado de polémica y corrupción ya que no se difundió la convocatoria de participación para el público en general y los ciudadanos se enteraron a inicios de 2014 sólo a través de la prensa local, que informó sobre el proyecto que resultó ganador. El objetivo de la revitalización buscaba posicionar a la Macroplaza como 
"destino cultural, recreativo y turístico, una zona de encuentro, de identidad e inclusión social" (Eco Político, 2016). Sin embargo, la comunidad de arquitectos del estado, al conocer el proyecto y enterarse de que supuestamente se sometió a concurso público (que no fue difundido), alzó la voz por los malos manejos y por la imposición del proyecto, el cual, además, no se adecuaba a las especificaciones sugeridas. El proyecto contemplaba la construcción de pasos peatonales elevados y la destrucción de las áreas verdes presentes en la plaza. La presión del gremio fue tanta, que la intención de revitalizar la Macroplaza terminó por diluirse.

El proyecto Monterrey Histórico fue un programa impulsado por el sector público municipal, bajo la administración de la entonces alcaldesa Margarita Arellanes. Contemplaba realizar un programa integral de mejora y rehabilitación urbana, tanto de viviendas como de espacios públicos, para fomentar el desarrollo cultural del lugar. Sin embargo, el ambicioso proyecto sólo quedó en el papel (Hernández, 2016).

El proyecto Paseo Ocampo consistía en el mejoramiento urbano de dicha avenida, traducido en ampliación de banquetas, carril exclusivo para transporte urbano y ciclovías. Se realizó desde iniciativas públicas y privadas, con una inversión de 53 millones de pesos.

El Festival Callegenera surgió en 2010 y busca dar espacio a expresiones artísticas culturales locales e internacionales. Realizado anualmente desde 2011, el festival pretende "conjuntar las expresiones urbanas que hay en la ciudad" (Portal Político, 2011) para visibilizar otras maneras de hacer arte, como a través del grafiti urbano.

Si bien en todos y cada uno de estos proyectos se pueden intuir buenas intenciones, muchos de ellos no se desarrollaron ni se consolidaron, por lo que sería conveniente estudiar la razón de ello.

\section{b) Proyectos de rehabilitaciones arquitectónicas}

Los proyectos clasificados como rehabilitaciones arquitectónicas son aquellos que han restaurado, remodelado o reconvertido un edificio existente en el centro de la ciudad para darle un nuevo uso. El primer proyecto de reconversión arquitectónica fue Urbania, construido sobre el antiguo edificio Monterrey, cuyo objetivo fue apostar por un nuevo modelo de vida insertado en la calle comercial Morelos.

Otros proyectos que contribuyen a fomentar y preservar el patrimonio cultural del centro de Monterrey son los que corresponden a restauraciones arquitectónicas. Es el caso de la restauración de la pintura mural del 
Templo de Nuestra Señora de Dolores, construido en 1909 y declarado patrimonio cultural del Estado de Nuevo León. O bien, la restauración de la fachada principal y del conjunto escultórico de la Basílica de la Purísima. Estas intervenciones se realizaron con dinero público, a través del Conarte y del Consejo Nacional para la Cultura y las Artes (Conaculta), entre otros organismos públicos.

\section{c) Proyectos de nueva construcción}

Los proyectos de nueva construcción apuestan por desarrollar el sector inmobiliario en el centro a través de edificios verticales de usos mixtos. Su desarrollo empezó a inicios de 2010, siendo uno de los proyectos pioneros el edificio de La Capital, ubicado a un lado del río Santa Lucía. Desde ese momento comenzaron a multiplicarse estas edificaciones en el centro de Monterrey, las cuales contrastan con la baja densidad del centro.

\section{d) Proyectos de participación comunitaria}

Mientras algunas iniciativas procedentes de la administración mantienen una política de renovación y modernización orientada al mercado inmobiliario, los proyectos con participación ciudadana muestran la necesidad de mejorar el entorno, dinamizando y activando la cultura local, y tomando los recursos disponibles a su alcance. Los proyectos de participación comunitaria son aquellos que surgen a partir de iniciativas ciudadanas. Ramírez (2009, p. 74) lo define bien:

Cierto grupo de la sociedad, al sentirse excluido de la ideología de la oferta cultural, toma la iniciativa y promueve proyectos autogestivos que llenan los vacíos que las políticas culturales, corporativas y públicas no han atendido, a costa de su propia economía.

Dentro de esa tipología se encuentra la regeneración del Barrio del Nejayote, impulsada por el colectivo Caminando en mi Barrio, integrado por vecinos del área, quienes, a través de la mejora estética de sus aceras, usando la técnica del trencadís, han revalorizado una zona que durante años arrastró un fuerte estigma social, mejorando su imagen urbana y atrayendo turismo y creación de actividades culturales (Díaz-Fernández y Ledesma-Gómez, 2018). 
Algo similar sucede en el Barrio de la Luz, lugar donde se han asentado artistas y creadores, generando una comunidad artística en el centro que ofrece actividades culturales a vecinos, además de promover la cohesión social entre ellos. Si bien aún no se visibilizan regeneraciones tangibles en el barrio, se ha creado una red de colaboraciones entre espacios culturales y activistas, lo que nos habla de un barrio cohesionado.

Otro de los proyectos impulsados por la sociedad civil es el Parque Ciudadano, el cual surgió a finales de 2014 gracias a los ciudadanos que lograron detener el inicio de la construcción de un estacionamiento para trabajadores del sector público en una de las pocas áreas verdes existentes en el centro. La falta de espacios verdes, junto con el valor ecológico del solar por tener más de 30 árboles de la región, fueron motivo suficiente para que ciudadanos, con apoyo de la academia, se manifestaran en contra de la obra, argumentando que en lugar del estacionamiento podría crearse un espacio público. Para impedir el inicio de la obra, los ciudadanos "okuparon" el solar durante 14 días ininterrumpidos en uno de los inviernos más crudos de la ciudad. La presión ciudadana, tanto presencial como a través de las redes sociales, logró detener permanentemente la construcción del estacionamiento. Durante el tiempo de negociación ciudadana, activistas y residentes utilizaron el área como espacio público, organizándose para llevar a cabo acciones de limpieza y mantenimiento. Después de dos años, concluida la negociación, se convocó a concurso público el diseño del parque, y fue a finales de 2017 cuando el sector público, con el proyecto ganador, comenzó la construcción del parque. Para ello se utilizó el 1\% del presupuesto asignado al estacionamiento. Es en abril de 2018 cuando el Parque Ciudadano fue finalmente inaugurado, mostrando que la ciudadanía es capaz de ganar batallas.

Esta revisión del proceso de regeneración urbana, junto con la breve descripción respecto a las políticas culturales en Monterrey, nos permite entender que, aunque hay buenas intenciones por parte del sector público y del privado, muchos de los proyectos realizados no terminan por consolidarse, mientras que, a su vez, la ciudadanía ha demostrado capacidad resiliente para enfrentar estas vicisitudes.

\section{Resultados: radiografía del centro de Monterrey desde el mapeo cultural}

La riqueza cultural del territorio se identifica a través de los recursos culturales orientados a las infraestructuras que brindan atributos locales, natura- 
les, arquitectónicos y patrimoniales a los habitantes del lugar, ayudando a reconocer los recursos culturales para poder desarrollar políticas enfocadas a mejorar el impulso de estas infraestructuras (Ghilardi, 2003).

Se mapearon los recursos culturales tangibles del centro clasificándolos en las siguientes categorías: centros educativos, espacio público, espacios culturales independientes (ECI), equipamientos culturales y elementos simbólico-patrimoniales.

\section{Mapa 2}

Mapa cultural del centro de Monterrey

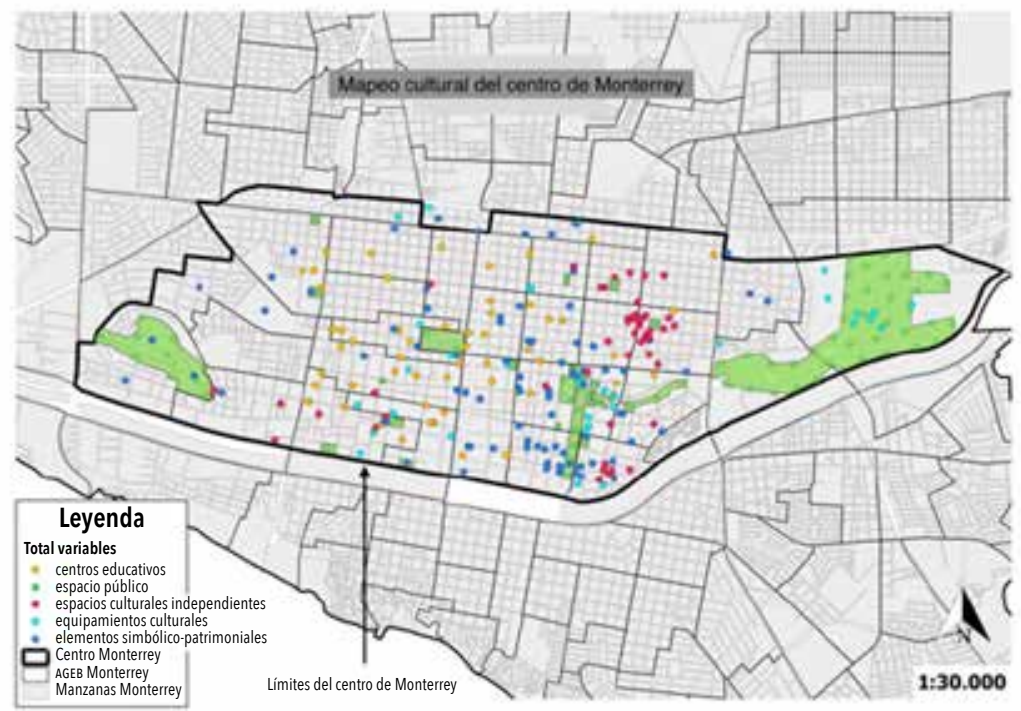

Fuente: Elaborado por Helena Grau y Fabiola Garza.

Con los espacios públicos pasa algo similar que con las áreas verdes: existen, pero no están enlazados, por lo que no promueven la peatonalización de la zona.

Los espacios culturales independientes (ECI) son sitios culturales y educativos, como talleres, galerías, cafés, lugares de coworking, tiendas y talleres tradicionales (como los de elaboración de piñatas), y talleres de joyería y relojería. Surgieron a finales de 2012, comenzando a multiplicarse en los barrios de la Luz, el Chorro y el Barrio Antiguo. Es interesante que 
la población que habita en estos lugares es gente creativa y comprometida con el sector cultural y el arte, lo que podría indicarnos el inicio del arraigo de una clase creativa en la zona (Florida, 2005). Resulta curioso el caso del Barrio de la Luz, el cual, a partir de estas iniciativas, ahora recupera su identidad pues históricamente ha sido un barrio de artes y oficios.

Los elementos simbólico-patrimoniales son monumentos históricos omnipresentes en gran cantidad en el centro. La mayor parte se localiza en la franja media, con una distribución bastante buena entre ellos. Resulta interesante ver estos bienes culturales mapeados, ya que, a nivel de calle, su presencia resulta casi imperceptible. Sin embargo, muchos de ellos se encuentran en estado precario, sin recibir mantenimiento alguno; tal es el caso del Arco de la Independencia, ubicado en el cruce de las avenidas Pino Suárez y Madero.

\section{Mapa 3}

Intensidad cultural en centro de Monterrey

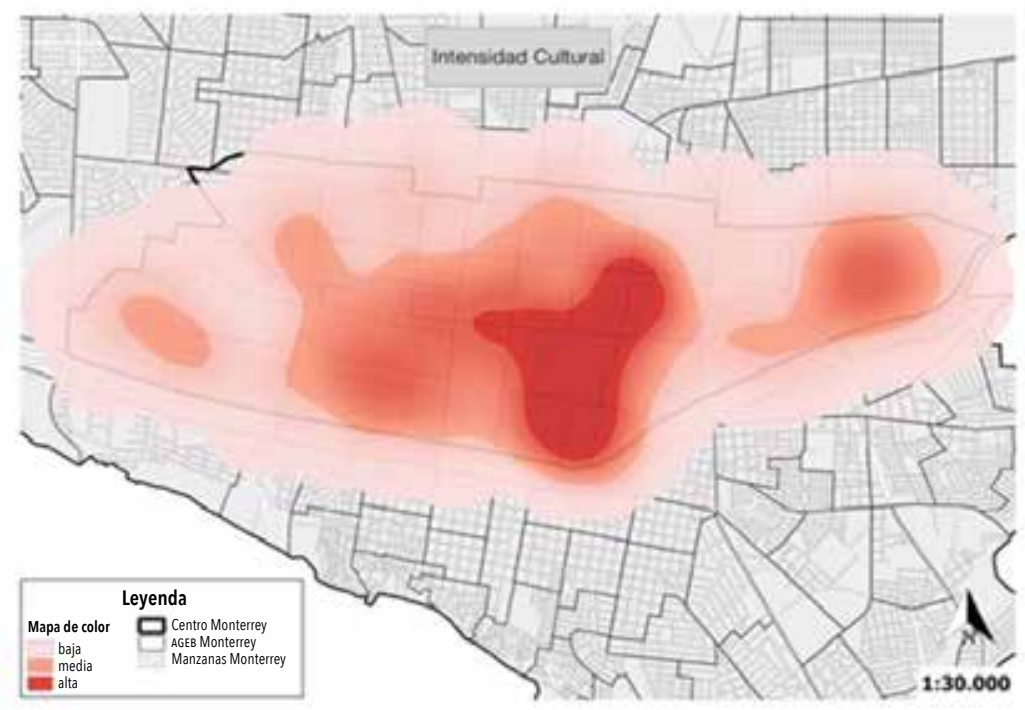

Fuente: Elaborado por Helena Grau. 


\section{El complemento al mapeo cultural: las narrativas sociourbanísticas}

A continuación, se presentan resultados a modo de narrativas urbanísticas que definen al centro de Monterrey. Como se ha mencionado anteriormente, las narrativas surgen de entrevistas realizadas a actores clave para visibilizar su percepción en torno a las estrategias urbanísticas implementadas y las formas en que la cultura se visibiliza como elemento de transformación y regeneración del centro.

\section{El juicio: las problemáticas urbanas y su origen}

Una narrativa en común manifiesta que el principal problema del centro es su estado de abandono. La explicación al problema tiene un trasfondo sociolegal. Muchos de los propietarios de los inmuebles del centro mueren sin hacer testamento, lo que genera un vacío legal pues los inmuebles quedan intestados; en pocas palabras, no tienen dueño. El proceso legal a seguir cuando un inmueble se encuentra intestado es largo y costoso, motivo por el cual se ha vuelto una escena común ver inmuebles en este estado. Junto con tal problemática, el centro se percibe como un lugar no habitable.

Una narrativa que contrasta con la anterior, específicamente de representantes de colectivos, indica que el problema es de percepción y no realmente de abandono. Activistas e investigadores concuerdan en que sí hay abandono, pero argumentan que este fenómeno no es generalizado ni extrapolable a todo el territorio del centro. Hay que recordar que el centro se compone de muchos centros; con esto nos referimos a que dentro de los límites de su territorio existe gran diversidad de usos de suelo, junto con dinámicas sociales que caracterizan y dan identidad y vida propia a cada zona o barrio. Por ejemplo, dentro de las zonas que destacan podemos mencionar al polígono declarado como centro histórico: el Barrio Antiguo. También la zona comercial de la calle Morelos, junto con el área hotelera que se encuentra a su alrededor. Está también el Barrio de la Purísima, sede de oficinas y negocios, junto con el Barrio del Mediterráneo, así como el Barrio de la Luz. Se argumenta que la percepción de abandono se produce a partir de la falta de mantenimiento urbano por parte del sector público. Otras problemáticas vinculadas son la falta de equipamientos públicos, zonas culturales, espacios de recreación, áreas verdes y servicios básicos. La ausencia de complejidad urbana es una de las mayores limitantes para la constitución de un centro compacto, accesible y cohesionado, siendo esta variable una de las causas que podría estar frenando dinámicas que promuevan la calidad urbana. 
Como narrativa complementaria, es interesante la postura del sector público municipal y del sector privado, quienes manifiestan que el abandono y la falta de proyectos de renovación urbana en el centro se deben a que actualmente los incentivos económicos y fiscales para crear desarrollos inmobiliarios no se orientan al centro, prefiriendo apostar por desarrollos en otros sectores del área metropolitana, como en el municipio vecino de San Pedro Garza García. A esta problemática se le suma el proceso legal para construir o remodelar un inmueble, donde los trámites son largos y complicados, lo que no es atractivo para el sector privado. En esa línea, la narrativa del actor del sector público municipal es que la autoridad se enfoca exclusivamente en "inventar" normas y estudios que no se necesitan para la propiedad privada en el centro, lo que deviene en un desincentivo para invertir en la zona.

Si yo quiero hacer una vivienda de cierto tipo y quiero ponerle a mi casa tres pisos, pero voy a la Secretaría de Desarrollo Urbano, me van a atorar dos años con el trámite y me van a pedir dinero y estudios como si fuera una torre muy complicada $[\ldots]$ te matan con la pura tramitología y normatividad [Exdirector de ImplancMty].

Las causas y la responsabilidad de las problemáticas asociadas a la vivienda y al abandono en el centro recaen en el gobierno municipal y en el estatal, por ser ellos los responsables de crear políticas de construcción y mantenimiento junto con estrategias de mejora; pero éstas han tendido a favorecer la especulación urbanística y, además, están comenzando a producir fenómenos de gentrificación.

El sector privado también tiene responsabilidad porque crea inmuebles que tienden a promover el mercado inmobiliario, produciendo fenómenos como la especulación urbanística, más que a promover unificación social. La especulación se manifiesta en la compraventa de terrenos o inmuebles en mal estado para remodelarlos y obtener un mayor beneficio económico con su venta. Con la venta se genera la gentrificación, ya que comienzan a transformar el nivel de renta, tendiendo al desplazamiento de la población local. Dicho fenómeno ya es visible en el centro de Monterrey, en donde comienzan a transformarse antiguos inmuebles abandonados en galerías de arte o cafés culturales, provocando el desplazamiento de la población; tal fenómeno ha sido estudiado en ciudades latinoamericanas por Carrión (2007), quien lo denomina boutiquización, pues el uso de suelo residencial se modifica a comercial. Esto se aprecia en barrios latinoamericanos como, por ejemplo, el Barrio de Getsemaní en Cartagena de Indias, Colombia (Rius-Ulldemolins y Posso, 2016). 
Por la misma línea, el sector público apuesta por la redensificación, apoyando al sector privado con políticas y cambios de uso de suelo para la construcción de edificios verticales de usos mixtos. Tal fenómeno, que Insulza-Contardo (2012) define como latinogentrificación, sustituye la tipología de inmuebles existentes por edificaciones de altura, caso que ha sido estudiado en Santiago de Chile, y se da en el centro de Monterrey a través de proyectos como La Capital, Pabellón M o El Semillero. Sin embargo, en el caso del centro de Monterrey, la falta de políticas públicas urbanas y de incentivos que permitan el acceso a la vivienda son factores que frenan su ocupación, ya que, además, los proyectos se orientan hacia la población con nivel medio o medio-alto de renta.

La destrucción del patrimonio arquitectónico es otra problemática grave y preocupante que sucede día tras día en el centro. Ahí existen edificaciones de alto valor patrimonial, inmuebles que datan del siglo XVIII, XIX y principios del XX, además de edificios representativos del movimiento moderno y art decó, que cuentan la historia de la ciudad. La falta de conocimiento del valor patrimonial por parte de los dueños de los inmuebles es la mayor amenaza contra la historia y la memoria de la ciudad, ya que atenta contra su preservación, eliminando de manera arbitraria su pasado urbano y produciendo procesos de gentrificación simbólica; este concepto, estudiado por Janoschka y Sequera (2014) y Delgadillo (2009), la define como aquella orientada a producir políticas de intervención de patrimonio arquitectónico exclusivo para turistas y élites sociales, generando la expulsión de los vendedores ambulantes. Tal fenómeno es recurrente en el centro de Monterrey: se orienta su regeneración hacia lo que anteriormente denominamos "alta cultura", produciendo, además, lo que Vargas Llosa (2012) define como banalización de la cultura, pues se reduce a satisfacer las necesidades de ocio y recreación, lo que es visible en las atracciones turísticas creadas en la ciudad, como el Paseo Santa Lucía y el Museo Horno 3 del Parque Fundidora.

\section{Radiografía cultural: causas y acciones}

En este apartado se realiza una radiografía y un análisis de lo encontrado dentro del clúster cultural. En el área donde se ubica, la cual corresponde al polígono del Barrio Antiguo, la zona de la Macroplaza y el Barrio de la Luz, existen dinámicas económicas, sociales y culturales heterogéneas. Por una parte, hay usos de suelo comerciales, de servicios y culturales; estos últimos están dedicados principalmente al turismo y al ocio nocturno, como es el caso del Barrio Antiguo, polígono orientado a satisfacer el ocio nocturno 
del centro de la ciudad. Conocido como el centro histórico, desde hace poco más de veinte años se ha convertido en el polo de ocio nocturno del centro, originando que el sector público y el privado produzcan actuaciones urbanísticas enfocadas en mejorar su imagen urbana a través de intervenciones estéticas que, como bien apunta Prieto González (2015), tienden más hacia el embellecimiento urbano que a generar transformaciones urbanas contundentes. El Barrio Antiguo también se caracteriza por ser sede de la escena cultural y bohemia, con presencia de artistas que tienen sus talleres de arte en el barrio, junto con comercios destinados a la venta de antigüedades, creación de mobiliario o espacios que promueven el arte y la cultura.

Desde la proyección de su primera regeneración en 1994, el Barrio Antiguo se ha convertido en zona de ocio y entretenimiento (Cabrales, 2000). Comenzaron a aparecer antros, bares y clubes nocturnos que, además de alterar el contexto arquitectónico al modificar construcciones antiguas, inciden de manera negativa en las formas de vida de los residentes debido al ruido, la basura y el congestionamiento vial y peatonal producidos a altas horas de la noche.

La violencia ligada al narcotráfico vivida en Monterrey en el periodo más álgido (2007-2013), junto con el atentado hacia uno de los bares de culto más representativos de la ciudad en 2011 -el Café Iguana-, provocaron un declive considerable en las formas de vida y en la actividad nocturna del barrio por el temor a enfrentar nuevas situaciones de riesgo. Un dato necesario de mencionar es la decisión del dueño del Café Iguana de dejar las marcas de balazos en la fachada como símbolo de resistencia y memoria de lo ocurrido.

Barrio Antiguo siempre ha tenido un toque cultural, pero en la última década tuvo un apogeo de antros; tenía una vida nocturna muy importante que fue afectada por el periodo de extrema violencia de 2007 a 2013; hubo un proceso de abandono de la zona muy grande [...] ahora ya se recuperó un poco y está volviendo a haber vida de negocios [presidente de Vecinos Barrio Antiguo].

Una de las desventajas de la actividad nocturna del barrio es que, además de provocar descontento entre los residentes locales, también ha originado el desplazamiento de la población y, con ello, la pérdida de identidad. Los establecimientos nocturnos, además de alterar el contexto urbano y poblacional, han cambiado la morfología arquitectónica del lugar al modificar las construcciones declaradas patrimonio arquitectónico, las cuales, a pesar de estar catalogadas por instituciones públicas de protección del patrimonio, como el Instituto Nacional de Antropología e Historia (INAH) y el Ins- 
tituto Nacional de Bellas Artes (INBA), no han sido protegidas para evitar su modificación y/o destrucción. Este hecho es recurrente entre las instituciones culturales en Monterrey.

\section{Fotografía 2}

El Barrio Antiguo. Café Iguana con los impactos de bala en su fachada

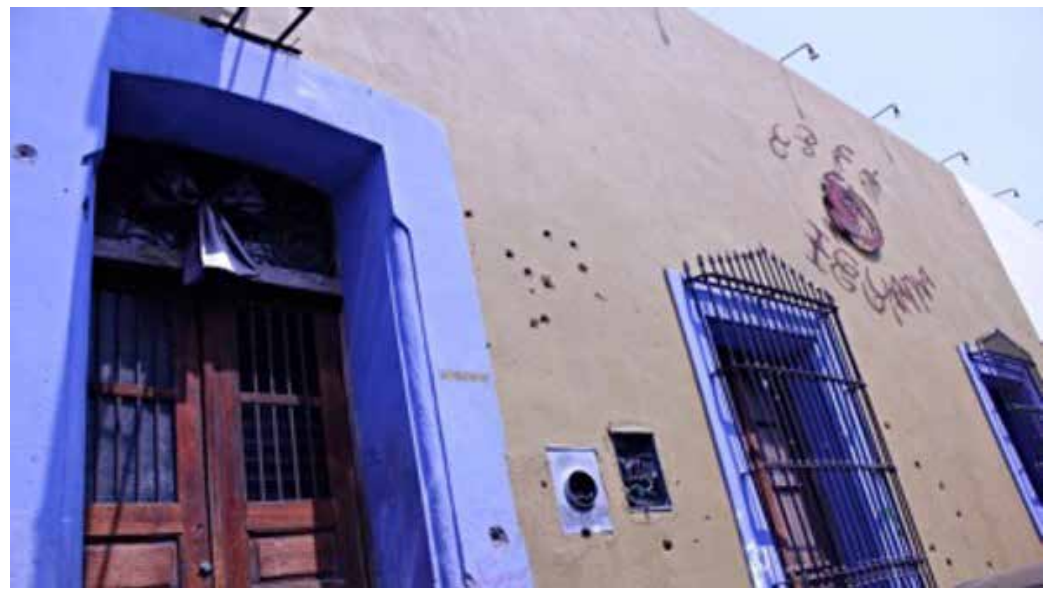

Fuente: Fotografía de Fabiola Garza-Rodríguez.

Como ejemplo, podemos mencionar el caso del Mercado Barrio Antiguo. En el verano de 2014 se anunció la creación de un mercado gourmet en el polígono. Para su ubicación, se eligieron dos predios colindantes, los cuales se encuentran ocupados por edificaciones antiguas. Una de ellas (la casona ubicada en la calle Padre Mier) está protegida por ser un inmueble de inicios del siglo XX y está inscrita en el Catálogo de inmuebles con valor histórico y artístico de la zona protegida del Barrio Antiguo, que data de 2013; además del valor patrimonial del inmueble, se encuentra el valor arquitectónico, ya que cuenta con sistemas constructivos de la arquitectura norestense, como techumbre de vigas de madera y materiales vernáculos propios de la región como la piedra de sillar. La polémica inició cuando arquitectos, ciudadanos y promotores culturales se enteraron a través de noticias de prensa (Mendieta Sánchez, 2015) que, para realizar el proyecto, se demolerían los inmuebles en su totalidad, sin realizar un peritaje previo que lo justificara, como fallas estructurales u otra causa de rigor que comprobaría la necesidad de su inminente demolición. 
El INAH es quien realiza los catálogos de bienes muebles e inmuebles con valor patrimonial, además de ser la institución pública encargada de velar por la protección del patrimonio cultural. Sin embargo, de manera contradictoria, es a la vez quien aprueba la demolición de construcciones protegidas, al menos en el estado de Nuevo León (Mendoza Lemus, 2016). Fue el INAH quien autorizó los permisos correspondientes para demoler las dos casonas referidas para dar lugar al nuevo mercado (Mendoza Lemus, 2016). Sin embargo, al buscar responsables se entra en una espiral de la que no se encuentra salida, porque los vecinos manifiestan que hubo corrupción para la creación de este mercado al no respetarse el Reglamento de Conservación y Preservación del barrio (Mendieta Sánchez, 2015). Por otra parte, un delegado público municipal alegaba que se estaba cumpliendo con la ley y que el inversionista privado seguía los requerimientos arquitectónicos establecidos por el INAH (Mendieta Sánchez, 2015).

Otra polémica es la sucedida a mediados de noviembre de 2016 en el predio conocido como Casa Allende, ubicado en la calle homónima esquina con Escobedo. Comenzó cuando un ciudadano concientizado sobre la importancia de proteger el patrimonio de la ciudad fue testigo de la demolición de un inmueble del siglo XIX. El ciudadano decidió exponerlo en las redes sociales, donde se sumaron arquitectos, restauradores y sociedad civil, quienes decidieron hacer una manifestación frente a la casa en demolición. Reunidos ahí, se argumentó que el inmueble se encontraba dentro del catálogo de patrimonio del INAH; sin embargo, ya se había demolido en 70\%. El caso estuvo cargado de polémica ya que la delegada del INAH alegó que ella no dio el permiso de demolición, pero algunas fuentes mostraron el oficio firmado por ella. En defensa propia, la delegada anunció que no recordaba haberlo firmado y que posiblemente "estaba dormida" cuando lo hizo (Villegas, 2016). Parece un episodio de algún programa cómico, pero no lo es. Es lo que sucede con las instituciones culturales y el patrimonio de Monterrey. Estas situaciones provocan desconfianza en las instituciones, ya que la sociedad civil -y con justa razón- no se puede explicar que el organismo público encargado de salvaguardar el patrimonio arquitectónico y cultural de la ciudad permitió su destrucción. Como defensa, el INAH argumenta que, al ser los inmuebles privados, no puede impedir que los dueños realicen intervenciones en éstos. Sin embargo, ello nos habla de un fallo importante en la creación de políticas de protección al patrimonio, ya que la misión del INAH es protegerlo y, a su vez, concientizar a los propietarios de lo que poseen. Hace falta replantearse de manera profunda quiénes están al frente de estas instituciones y las estrategias de protección o las formas de mejorar normativas y sanciones. 
Dentro del clúster cultural se encuentra también el Barrio de la Luz, polígono orientado a las artes y oficios del Monterrey antiguo, en donde anteriormente había talleres y espacios destinados a ofrecer servicios como sastrerías, carpinterías, joyerías, talleres de arte, ferreterías, zapaterías, entre otros. Hoy en día, aún permanecen algunos de estos establecimientos, lo que refuerza su identidad como barrio cultural. Es el caso, por ejemplo, de una ferretería que tiene más de treinta años en el barrio, de un taller relojero o el taller de piñatas. La permanencia y conservación de los pequeños comercios es un fenómeno que se reconoce como parte del barrio, dándole valor añadido a su tejido sociourbano, ya que refuerza su identidad como área destinada a ofrecer servicios culturales específicos.

Las entrevistas nos permiten saber que el Barrio de la Luz ha sido uno de los pocos que no ha perdido población. A inicios de 2013, en el polígono se empezó a dar un fenómeno interesante: la aparición de espacios culturales independientes destinados a talleres de arte, galerías, librerías, escuelas artísticas y cafés culturales, que surgieron desde la misma sociedad civil, apareciendo de manera autónoma para llenar el gap cultural que el sector público había dejado en la ciudad y, de paso, promover la descentralización de la cultura. La proliferación de espacios culturales ha desencadenado efectos positivos tanto en la población como en el tejido urbano del lugar, ya que, al estar agrupados en un determinado vecindario, las redes colaborativas entre artistas, dueños, habitantes y ocupantes de los espacios han reforzado la identidad colectiva del barrio mediante el arte y la cultura. Las redes sociales contribuyen en mantener la vitalidad de la zona, ya que a través de ellas se publican eventos, talleres, conferencias o festivales que tienen lugar en el barrio, lo que permite una mayor difusión y reconocimiento de las actividades en el lugar. La escala de barrio posibilita que haya un reconocimiento entre los habitantes, generando dinámicas de confianza. Algo similar sucedió en el barrio de Savamala en Serbia, en donde las iniciativas culturales independientes han logrado impulsar y revitalizar culturalmente esa área a partir de un enfoque bottom-up y sin planeación alguna (Lazarevič, Koružnjak y Devetaković, 2016). Ésta también puede ser un área de oportunidad de regeneración integral en el centro de Monterrey.

Los proyectos que surgen desde iniciativas de abajo hacia arriba están transformando la estética del barrio y refuerzan su sentido de pertenencia con actividades que involucran la identidad local. Han sido realizados por residentes locales, pero es importante que los sectores público y privado colaboren, ya que es la forma de lograr transformaciones urbanas que regeneren no sólo el centro, sino el resto de la ciudad. Este fenómeno ya se ha 
observado tanto en otras ciudades latinoamericanas (Parque Berrio en Medellín, Colombia), como europeas (Barrio del Raval, en Barcelona).

\section{Potencialidades de transformación sociourbanística y cultural (o el rol de la cultura como elemento de transformación urbanística)}

Además de visibilizar los problemas del centro y abordar algunas de las estrategias de regeneración urbana implementadas, en la entrevista se preguntó de manera directa sobre el papel que la cultura puede jugar como elemento de transformación sociourbana que promueva dinámicas de sostenibilidad cultural. Se indagó sobre las potencialidades socioculturales del centro en términos tangibles, como el estado de los espacios públicos, recreacionales, patrimoniales y culturales. Se identificó una narrativa en común: la ciudad cuenta con buen potencial de estos espacios, pero hay una cantidad limitada de ellos, por lo que es necesario tomar ventaja de los elementos existentes (espacios públicos, monumentos históricos, museos e iniciativas culturales independientes) para producir y reforzar el contexto cultural de la zona. Esta tendencia narrativa, que coincide con el mapa cultural realizado, muestra la poca articulación entre los recursos culturales existentes, los cuales podrían ser elementos que se aprovecharan para conectar los nodos de interés cultural en el centro. Existen dos narrativas en cuanto a si existe una buena cobertura y distribución de los equipamientos culturales. Algunos entrevistados manifestaron que el centro cuenta con buena distribución de ellos, pero no son accesibles para toda la población, pues se concentran en determinadas zonas y están orientados a determinados círculos sociales. Otros coincidieron en que hace falta dotar al centro de más infraestructura cultural, ya que los espacios existentes son limitados.

Realmente hay muy pocos y están más pegados al Barrio Antiguo. Son los tres museos, pero son pocos; además de que están demasiado atomizados en ese punto porque están sobre la misma calle los tres y vas a uno, vas a otro y vas a otro y ya. Necesitan estar más separados [crítico arquitectónico].

Las preguntas finales sobre cómo el paradigma cultural puede ser usado para la planeación urbana y para plantear soluciones de mejora fueron pocas, pero precisas. Una de las narrativas principales argumenta la necesidad de crear más espacios públicos, ya que son lugares que permiten el entendimiento de la ciudad conforme a su sociedad (Borja y Muxi, 2003): ahí radica su importancia como conformador de identidad de un lugar, argumento 
que además se asocia a lo que definimos en un inicio como cultura. Otra narrativa solicita más intervenciones urbanas para reajustar las necesidades de la sociedad mediante proyectos relacionados de arquitectura social. Realizar proyectos a modo de acupuntura urbana también es una narrativa recurrente, en donde se reparen zonas del centro mediante intervenciones realizadas a escala micro. Otra narrativa argumenta la necesidad de ofrecer variedad cultural o a través de la promoción de movimientos artísticos.

\section{Consideraciones finales}

En los últimos años se ha reconocido la importancia de la cultura como elemento y motor del desarrollo urbano (Degen y García, 2012; Jeannotte, 2016), por lo que su uso como respuesta a problemáticas urbanas cada vez es más utilizado por el abanico de intervenciones que puede generar (Miles y Paddison, 2005; Zukin, 1995). El mapeo cultural es una técnica emergente de análisis que permite conocer la distribución geográfica de los recursos culturales en un determinado territorio. En se sentido, el centro de Monterrey es un espacio único en la ciudad en donde se reconocen distintas realidades. Esto hace referencia a las zonas en las que hay mayor actividad turística, otras en donde predomina lo cultural, o a los sectores de oficinas, e inclusive a las áreas en donde hay una mayor actividad habitacional.

La concentración de recursos culturales nos permite identificar un clúster cultural en el centro de Monterrey. Los clústeres de este tipo generan dinámicas microscópicas que producen cambios urbanos y sociales en la forma de vivir la ciudad (Lazarevič, Koružnjak y Devetaković, 2016), pudiendo ser el preámbulo de una transformación urbana orientada desde la sostenibilidad cultural. De ahí la importancia de reconocer su existencia en nuestro caso de estudio.

No parece que el sector público de manera deliberada haya apostado por la creación de un clúster cultural en el centro de Monterrey, al menos no existe una política que lo sustente al respecto. Sin embargo, por la localización de los equipamientos y la concentración de los recursos culturales, se ha generado un importante polo de aglomeración de cultura y turismo en la ciudad, creándose un clúster cultural de manera intencionada, pero a la vez, aleatoria. El discurso político actual está comenzando a prestar más atención a esta zona debido a los equipamientos ya existentes y a la presión mundial generada por reconocer la importancia de la cultura como motor de desarrollo (por ejemplo, a través de la Agenda 21 de la Cultura, la cual ya han adoptado muchas ciudades latinoamericanas). 
A pesar de la falta de proyectos contundentes que realmente revitalicen al centro por parte del sector público-privado, la zona cuenta con una alta riqueza cultural por la presencia de recursos culturales que existen dentro de sus límites; sin embargo, no se aprovechan de manera adecuada para generar transformaciones urbanas significativas y perdurables. Las prácticas artísticas y culturales en la ciudad deberían ser reconocidas como parte integral del desarrollo sostenible en forma de políticas culturales. Sería necesario replicar en el centro de Monterrey algunas estrategias que busquen soluciones adaptadas a cada área o barrio que lo conforma.

Las entrevistas han permitido constatar que el centro de Monterrey padece problemáticas urbanas como el abandono y la falta de infraestructura. A la vez, muestran la percepción de un grupo de entrevistados sobre lo que el sector público y el privado están realizando para su regeneración. Cuando se llevó a cabo la recolección de datos, se encontraron reflexiones similares entre los entrevistados; esto significa que es evidente que se necesita una política de intervención de manera urgente. Las entrevistas se consideran un proceso imprescindible para formular futuras propuestas para realizar mejoras e intervenciones urbanas, y deberían ser aplicadas a todos los contextos.

El sector público en Monterrey -de manera deliberada o no- ha abierto un camino de posibilidades de transformación que apuntan hacia la cultura. La fuerte inversión pública inyectada en los proyectos emblemáticos para mejorar trozos considerables del tejido urbano central es un gran paso en el camino hacia su transformación. Sin embargo, su principal defecto es la falta de continuidad en los proyectos, pues se construyen edificaciones, pero se piensa que por sí solas éstas regeneran el centro. En ese sentido, trabajar de la mano junto con los residentes locales se torna fundamental para mejorarlo de manera integral. El sector público deberá optar por realizar regeneraciones colaborativas entre los actores públicos, privados y sociedad civil (Healey, 2003). Las investigaciones demuestran que aprovechar estas conexiones produce mejores proyectos, ya que tienden a la retroalimentación de ideas y actuaciones, lo que resulta en un mejor entendimiento de lo que es una regeneración, y contribuyen a la aplicación de la cultura al proceso (Jung, Lee, Yap e Ineson, 2015).

Es necesario fortalecer los lazos y el diálogo entre los actores, así como utilizar el enfoque de la sostenibilidad cultural para ayudar a los gestores y planeadores urbanos a identificar los recursos culturales y buscar alternativas para su uso y potenciación en áreas estratégicas. Aplicar la sostenibilidad cultural al planeamiento urbano implica mirar a la ciudad como un ecosistema vivo, compuesto por recursos culturales que deben ser reconocidos y evaluados por la comunidad local antes de aplicar políticas 
de intervención. Se deben formar coaliciones y sistemas de acuerdos entre iniciativas y organizaciones que permitan potencializar la cultura hacia acciones de interés público, como políticas culturales que fomenten la mejora urbana a través de las artes, las actividades culturales, las organizaciones y las mejoras en cuanto a la infraestructura cultural.

\section{Bibliografía}

Alanís, R. (2014). Inauguran remodelación de la calle Morelos. Milenio, 19 de diciembre. Recuperado de http://www.milenio.com/politica/inauguranremodelacion-de-la-calle-morelos

Aparicio, C. E., Ortega, M. E. y Sandoval, E. (2011). La segregación socio-espacial en Monterrey a lo largo de su proceso de metropolización. Región y Sociedad, 23(52), 173-207. Recuperado de https://www. redalyc.org/articulo.oa?id $=10221416006$

Axelsson, R., Angelstam, P., Degerman, E., Teitelbaum, S., Andersson, K., Elbakidze, M. y Drotz, M. (2013). Social and cultural sustainability: Criteria, indicators, verifier variables for measurement and maps of visualization to support planning. AMBIO. Journal of the Human Environment, 42(2), 215-228. Recuperado de https://www.ncbi.nlm.nih. gov/pubmed/23475657

Balbo, M., Jordán, R. y Simioni, D. (2003). La ciudad inclusiva. Cuadernos de la CEPAL (Documento de trabajo, núm. 88). Santiago de Chile: Comisión Económica para América Latina y el Caribe. Recuperado de https://repositorio.cepal.org/bitstream/handle/11362/27814/ S2003002_es.pdf\%3Bjsessionid\%3D8341F69732A246CA611D816D59208F4A\%3Fsequence\%3D1

Bazant, J. (2012). Deterioro del centro histórico de las ciudades. ¿Es viable su reactivación? Tiempo y Espacio, 29, 61-68. Recuperado de http:// www.ubiobio.cl/miweb/webfile/media/222/Tiempo/2012/\%2329.03. pdf

Becerril-Padua, M. (2000). Policentrismo en las ciudades latinoamericanas. El caso de Santiago de Chile. Revista Theomai, 1, 1-23. Recuperado de https://www.redalyc.org/articulo.oa?id=12400108

Becker, A. y Müller, M. M. (2013). The securitization of urban space and the rescue of downtown Mexico city: Vision and practice. Latin American Perspectives, 40(2), 77-94. Recuperado de https://journals.sagepub. com/doi/abs/10.1177/0094582x12467762 
Bianchini, F. y Parkinson, M. (eds.). (1994). Cultural policy and urban regeneration: The west European experience. Manchester, Reino Unido: Manchester University Press.

Borja, J. y Muxi, Z. (2003). El espacio público: ciudad y ciudadanía. Barcelona, España: Electa.

Cabrales, L. F. (2000). La rehabilitación del barrio antiguo de Monterrey. En B. Klauke (coord.), México y sus perspectivas para el siglo XXI (pp. 217-241). Münster, Alemania: Universität Münster.

Carrión, F. (2007). Financiamiento de los centros históricos de América Latina y el Caribe. Quito, Ecuador: Flacso / Lincoln Institute of Land Policy.

Casas García, J. M. (2015). Imaginarios ininterrumpidos. Ensayo sobre el patrimonio inmueble perdido en Monterrey. Monterrey, Nuevo León, México: Consejo para la Cultura y las Artes de Nuevo León.

Casgrain, A. y Janoschka, M. (2013). Gentrificación y resistencia en las ciudades latinoamericanas: el ejemplo de Santiago de Chile. Andamios. Revista de Investigación Social, 10(22), 19-44. Recuperado de http://www.scielo.org.mx/scielo.php?script=sci_arttext\&pid= S1870-00632013000200003

Catálogo de inmuebles con valor histórico y artístico de la zona protegida Barrio Antiguo de Montrerrey (2013). Monterrey, Nuevo León, México: Gobierno del Estado de Nuevo León. Recuperado de https://issuu. com/adanroca/docs/130437396-catalogo-2013-de-inmueble

Cauchi-Santoro, R. (2016). Mapping community identity: Safeguarding the memories of a city's downtown core. City, Culture and Society, 7(1), 43-54. Recuperado de https://www.sciencedirect.com/science/article/ pii/S1877916615300114

Colantonio, A., Dixon, T., Ganser, R., Carpenter, J. y Ngombe, A. (2009). Measuring socially sustainable urban regeneration in Europe (Documento de Investigacion). Oxford, Reino Unido: Institute for Sustainable Development. Recuperado de http://oisd.brookes.ac.uk/sustainable comunities/resources/Social_Sustainability_and_Urban_Regeneration_ report.pdf

Degen, M. y García, M. (2012). The transformation of the Barcelona model: An analysis of culture, urban regeneration and governance. International Journal of Urban and Regional Research, 36(5), 1022-1038. Recuperado de https://onlinelibrary.wiley.com/doi/full/10.1111/j.1468-2427. 2012.01152.x

Delgadillo, V. M. (2009). Patrimonio urbano y turismo cultural en la Ciudad de México: las chinampas de Xochimilco y el Centro Histórico. An- 
damios. Revista de Investigación Social, 6(12), 69-94. Recuperado de https://www.redalyc.org/articulo.oa?id=62815957004

Díaz-Fernández, A. D. y Ledesma-Gómez, R. D. L. (2018). Un barrio de color: el diseño de un museo al aire libre mediante trencadís en El Nejayote. Panambi. Revista de Investigaciones Artísticas, 6, 55-74. Recuperado de https://revistas.uv.cl/index.php/Panambi/article/view/1137

Duque Franco, I. (2015). La cultura como estrategia de transformación y promoción urbana en Bogotá y Medellín. Revista de Geografía Norte Grande, 61, 25-43. Recuperado de https://scielo.conicyt.cl/scielo. php?script $=$ sci_arttext\&pid=S0718-34022015000200003

Duxbury, N. (2014). Cultural governance in sustainable cities. Kultur: Revista Interdisciplinária sobre la Cultura de la Ciutat, 1(1), 165-182. Recuperado de http://www.e-revistes.uji.es/index.php/kult-ur/article/ view/1252

Duxbury, N., Garrett-Petts, W. F. y MacLennan, D. (2015). Cultural mapping as cultural inquiry: Introduction to an emerging field of practice. En N. Duxbury, W. F. Garrett-Petts y D. MacLennan (coords.), Cultural mapping as cultural inquiry (pp. 19-60). Nueva York, NY: Routledge.

Eagleton, T. (2001). L'idea di cultura. Roma, Italia: Riuniti.

Eco Político. (2016). Gobierno de Rodrigo Medina invita a revitalización de la plaza. Eco Político. Recuperado en http://www.ecopolitico.com.mx/ gobierno-de-rodrigo-medina-invita-a-revitalizacion-de-la-macroplaza/ $\mathrm{amp} /$

El Horizonte. (2015). Arrancará Emporio con 10 proyectos. El Horizonte, 6 de marzo. Recuperado en http://www.elhorizonte.mx/seccion/arrancaraemporio-con-10-proyectos--/1606126

Evans, G. (2005). Measure for measure: Evaluating the evidence of culture's contribution to regeneration. Urban Studies, 42(5-6), 959-983. Recuperado de https://journals.sagepub.com/doi/10.1080/00420980500107102

Evans, G. y Foord, J. (2008). Cultural mapping and sustainable communities: Planning for the arts revisited. Cultural Trends, 17(2), 65-96. Recuperado de https://www.tandfonline.com/doi/ abs/10.1080/09548960802090634

Florida, R. (2005). Cities and the creative class. Nueva York / Londres: Routledge.

Freitas, R. (2016). Cultural mapping as a development tool. City, Culture and Society, 7(1), 9-16. Recuperado de https://www.sciencedirect.com/ science/article/pii/S1877916615300035

García, B. (2004). Cultural policy and urban regeneration in western European cities: Lessons from experience, prospects for the future. Lo- 
cal Economy, 19(4), 312-326. Recuperado de https://journals.sagepub. com/doi/pdf/10.1080/0269094042000286828

Garza-Rodríguez, F. R. (2014). Análisis urbano y estrategias para la reforma del centro de Monterrey (Tesis de maestría, Universitat Politècnica de Catalunya). Recuperado de https://upcommons.upc.edu/ handle $/ 2117 / 78082$

Garza-Rodríguez, F. R. (2015). Evaluación de indicadores socio-urbanos y estrategias de reforma para el centro de Monterrey. Ponencia presentada en el VII Seminario Internacional de Investigación en Urbanismo, Departament d' Urbanisme i Ordenació del Territori. Universitat Politècnica de Catalunya. Barcelona / Montevideo, junio.

Ghilardi, L. (2003). Cultural planning: An integrated approach to cultural development. Plats, Drivkraft, Samhällsprocess, 7, 14-18. Recuperado de http://samla.raa.se/xmlui/bitstream/handle/raa/68/9172093374. pdf\#page $=16$

Harvey, D. (1989). From managerialism to entrepreneurialism: The transformation in urban governance in late capitalism. Geografiska Annaler: Series B, Human Geography, 71(1), 3-17. Recuperado de https:// www.jstor.org/stable/490503?seq=1\#metadata_info_tab_contents

Hawkes, J. (2001). The fourth pillar of sustainability: Culture's essential role in public planning. Australia: Common Ground Publishing.

Healey, P. (2003). Collaborative planning in perspective. Planning Theory, 2(2), 101-123. Recuperado de https://journals.sagepub.com/ doi/10.1177/14730952030022002

Heath, S. C., Rabinovich, A. y Barreto, M. (2017). Putting identity into the community: Exploring the social dynamics of urban regeneration. European Journal of Social Psychology, 47(7), 855-866. Recuperado de https://onlinelibrary.wiley.com/doi/full/10.1002/ejsp.2296

Hernández, I. (2016). Olvidan regeneración del centro de Monterrey. El Horizonte, 28 de agosto, p. 1. Recuperado de http://www.elhorizonte.mx/ local/olvidan-regeneracion-del-centro-de-monterrey/1680472

Hora Cero. (2017). Acuerdan Conarte y Consejo NL elaboración de diagnóstico cultural. Hora Cero, 29 de mayo, p. 1. Recuperado de https://www.horacero.com.mx/nuevo-leon/acuerdan-conarte-y-consejonl-elaboracion-de-diagnostico-cultural/

Inzulza-Contardo, J. (2012). Latino gentrification? Focusing on physical and socioeconomic patterns of change in Latin American inner cities. Urban Studies, 49(10), 2085-2107. Recuperado de https://journals. sagepub.com/doi/abs/10.1177/0042098011423425 
Janoschka, M. y Sequera, J. (2014). Procesos de gentrificación y desplazamiento en América Latina, una perspectiva comparativista. En J. J. Michelini (ed.), Desafíos metropolitanos. Un diálogo entre Europa y América Latina (pp. 82-104). Madrid, España: Catarata.

Jeannotte, M. S. (2016). Story-telling about place: Engaging citizens in cultural mapping. City, Culture and Society, 7(1), 35-41. Recuperado de https://www.sciencedirect.com/science/article/pii/S1877916615000 466

Jones, P. y Evans, J. (2008). Urban regeneration in the UK: Theory and practice. Londres, Reino Unido: Sage.

Jung, T. H., Lee, J., Yap, M. H. e Ineson, E. M. (2015). The role of stakeholder collaboration in culture-led urban regeneration: A case study of the Gwangju proyect, Korea. Cities, 44, 29-39. Recuperado de https:// www.sciencedirect.com/science/article/pii/S0264275114002066

Kana, K. (2012). An experiment in urban regeneration using culture and art in Senba, Osaka's historic urban center, with a focus on the regeneration of urban space. City, Culture and Society, 3(2), 151-163. Recuperado de https://www.sciencedirect.com/science/article/pii/S1877916612000185

Kanai, M. y Ortega-Alcázar, I. (2009). The prospects for progressive culture-led urban regeneration in Latin America: Cases from Mexico City and Buenos Aires. International Journal of Urban and Regional Research, 33(2), 483-501. Recuperado de https://onlinelibrary.wiley.com/ doi/full/10.1111/j.1468-2427.2009.00865.x

Landry, C. (2005). Lineagues of the creative city. En S. Franke y E. Verhagen (eds.), Creativity and the city: How the creative economy changes the city (pp. 42-54). Róterdam, Países Bajos: NAI Publishers.

Landry, C. y Bianchini, F. (1995). The creative city (vol. 12). Londres, Reino Unido: Demos.

Lange-Valdés, C. (2018). Architecture as an apparatus of urban regeneration: 20 years of the Bilbao Guggenheim Museum. Bitácora Urbano Territorial, 28(2), 115-123. Recuperado de http://dx.doi.org/10.15446/ bitacora.v28n2.70153

Lazarevič, E. V., Koružnjak, A. B. y Devetaković, M. (2016). Culture design-led regeneration as a tool used to regenerate deprived areas. Belgrade-The Savamala quarter; reflections on an unplanned cultural zone. Energy and Buildings, 115(1), 3-10. Recuperado de https://www.science direct.com/science/article/pii/S0378778815002078

Ley de Patrimonio Cultural del Estado de Nuevo León (1991). Periódico Oficial del Estado de Nuevo León [con última reforma integrada publicada el 2 de diciembre de 2015], 23 de diciembre. Monterrey, Nuevo 
León, México. Recuperado de https://sic.cultura.gob.mx/documentos/ 1678.pdf

Lipovetsky, G. (1994). La era del vacio. Ensayos sobre el individualismo contemporáneo. Barcelona, España: Anagrama.

Mancillas Hinojosa, A. C. (2009). Guía de patrimonio arquitectónico de Nuevo León. Monterrey, Nuevo León, México: Conarte.

Markusen, A. y Schrock, G. (2006). The distinctive city: Divergent patterns in growth, hierarchy and specialisation. Urban Studies, 43(8), 1301-1323. Recuperado de https://journals.sagepub.com/ doi/10.1080/00420980600776392

Martínez, S. (2018). Reportan 5 mil muertes prematuras al año en Monterrey por mala calidad del aire. La Jornada, 7 de enero, p. 1. Recuperado de https://www.jornada.com.mx/2018/01/07/politica/008n1pol

Mendieta Sánchez, E. (2015). Descarta Implanc corrupción en Mercado Barrio Antiguo. Milenio, 13 de junio, p. 1. Recuperado de http://www. milenio.com/estados/descarta-implanc-corrupcion-mercado-barrioantiguo

Mendoza, G. (2015). Leyes culturales de NL, sin peso y archivadas. Milenio, 19 de julio, p. 1. Recuperado de http://www.milenio.com/cultura/ leyes-culturales-de-nl-sin-peso-y-archivadas

Mendoza Lemus, G. (2016). Avaló INAH demolición de casona protegida. Milenio, 16 de noviembre, p. 1. Recuperado de http://www.milenio. com/cultura/avalo-inah-demolicion-de-casona-protegida

Mendoza Lemus,G. (2017). El Tío, un ejemplo de demolición histórica en el centro de la ciudad. Milenio, 30 de julio, p. 1. Recuperado de http:// www.milenio.com/estados/el-tio-un-ejemplo-de-demolicion-historicaen-el-centro-de-la-ciudad

Mendoza Lemus, G. (2018). Pide ampliar el centro histórico de la ciudad más allá de Barrio Antiguo. Milenio, 20 de enero, p. 1. Recuperado de http://www.milenio.com/estados/pide-ampliar-centro-historico -ciudad-alla-barrio-antiguo

Miles, S. y Paddison, R. (2005). Introduction: The rise and rise of culture-led urban regeneration. Urban Studies, 42(5-6), 833-839. Recuperado de https://journals.sagepub.com/doi/10.1080/00420980500107508

Montgomery, J. (2003). Cultural quarters as mechanisms for urban regeneration. Part 1: Conceptualising cultural quarters. Planning, Practice and Research, 18(4), 293-306. Recuperado de https://www.tandfonline. com/doi/abs/10.1080/1561426042000215614

Moreno, R. y Contreras, C. (2010). La ciudad del conocimiento: entre slogans y realidades. En L. Palacios (ed.), Cuando México enfrenta la 
globalización. Permanencias y cambios en el área metropolitana de Monterrey (pp. 131-152). Monterrey, Nuevo León, México: Universidad Autónoma de Nuevo León.

Papachristou, I. y Rosas-Casals, M. (2018). Cities and quality of life. Quantitative modeling of the emergence of the happiness field in urban studies. Cities, 88, 191-208. Recuperado de https://www.sciencedirect. com/science/article/pii/S026427511730940X

Park, S. H. (2016). Can we implant an artist community? A reflection on government-led cultural districts in Korea. Cities, 56, 172-179. Recuperado de https://www.sciencedirect.com/science/article/pii/ S0264275115001365

Pastak, I. y Kährik, A. (2016). The impacts of culture-led flagship projects on local communities in the context of post-socialist Tallinn. Sociologicky Casopis. Czech Sociological Review, 52(6), 963-990. Recuperado de http://cejsh.icm.edu.pl/cejsh/element/bwmeta1.element. $332 \mathrm{c} 58 \mathrm{e} 2-8 \mathrm{f} 72-4001-9049-\mathrm{d} 211 \mathrm{~b} 7 \mathrm{cfda} 0 \mathrm{e}$

Peck, J. (2005). Struggling with the creative class. International Journal of Urban and Regional Research, 29(4), 740-770. Recuperado de https:// onlinelibrary.wiley.com/doi/full/10.1111/j.1468-2427.2005.00620.x

Portal Politico. (2011). Por primera vez se realiza el Festival de Expresiones Urbanas Callegenera, un foro para la cultura popular. Portal Politico, 4 de junio, p. 1. Recuperado de https://www.portalpolitico.tv/ cultura/por-primera-vez-se-realiza-el-festival-de-expresiones-urbanascallegenera-un-foro-para-la

Prieto González, J. M. (2010). La consolidación del Monterrey imaginario en el contexto de la globalización: macroproyectos urbanos. Frontera Norte, 23(45), 163-192. Recuperado de http://www.scielo.org.mx/ scielo.php?script $=$ sci_arttext\&pid=S0187-73722011000100006

Prieto González, J. M. (2013). Monterrey como Estridentópolis: vigencia del ideal urbano de la vanguardia histórica mexicana. Palapa, 4(1), 17-31. Recuperado de http://revistasacademicas.ucol.mx/index.php/ palapa/article/view/101

Prieto González, J. M. (2014). Patrimonio moderno y cultura arquitectónica en Monterrey: claves de un desencuentro. Monterrey, Nuevo León, México: Fondo Editorial de Nuevo León / Universidad Autónoma de Nuevo León.

Prieto González, J. M. (2015). Lo humilde en un contexto de grandeza: desafíos que enfrenta la regeneración de Barrio Antiguo en Monterrey (Nuevo León). Contexto, 10(12), 11-28. Recuperado de http://contexto. uanl.mx/index.php/contexto/article/view/83 
Programa Especial de Cultura. (2017). Programa Especial de Cultura. Monterrey, Nuevo León, México: Gobierno del Estado de Nuevo León. Recuperado de http://www.nl.gob.mx/sites/default/files/programa_de_ cultura.pdf

Ramírez, E. (2009). El triunfo de la cultura: uso político y económico de la cultura en Monterrey. Monterrey, Nuevo León, México: Fondo Editorial de Nuevo León.

Reglamento del Barrio Antiguo de Monterrey. (1993). Periódico Oficial del Estado de Nuevo León, 15 de septiembre. Monterrey, Nuevo León, México. Recuperado de https://legislacion.vlex.com.mx/vid/reglamentobarrio-antiguo-monterrey-575239154

Rius-Ulldemolins, J. y Posso, L. (2016). Cultura, transformación urbana y empoderamiento ciudadano frente a la gentrificación: comparación entre el caso de Getsemaní (Cartagena de Indias) y el Raval (Barcelona). Eure. Revista Latinoamericana de Estudios Urbanos Regionales, 42(126), 97122. Recuperado de https://dialnet.unirioja.es/servlet/articulo?codigo= 5819785

Roberts, P., Sykes, H. y Granger, R. (eds.). (2016). Urban regeneration. Nueva York, NY: Sage.

Rojas, E. (2004). Volver al centro: la recuperación de áreas urbanas centrales. Nueva York, NY: Banco Interamericano de Desarrollo, Departamento de Desarrollo Sostenible.

Rueda, S., Cuchí, A., De Cáceres, R. y Brau, L. (2012). El urbanismo ecológico. Su aplicación en el diseño de un ecobarrio en Figueres. Barcelona, España: Agencia de Ecología Urbana de Barcelona.

Soini, K. y Birkeland, I. (2014). Exploring the scientific discourse on cultural sustainability. Geoforum, 51, 213-223. Recuperación de https:// www.sciencedirect.com/science/article/pii/S0016718513002558

Stern, M. J. y Seifert, S. C. (2010). Cultural clusters: The implications of cultural assets agglomeration for neighborhood revitalization. Journal of Planning Education and Research, 29(3), 262-279. Recuperado de https://journals.sagepub.com/doi/10.1177/0739456X09358555

Tallon, A. (2013). Urban regeneration in the UK. Londres, Reino Unido: Routledge.

Tavano Blessi, G., Tremlay, D. G., Sandri, M. y Pilati, T. (2012). New trajectories in urban regeneration processes: Cultural capital as source of human and social capital accumulation. Evidence from the case of Tohu in Montreal. Cities, 29(6), 397-407. Recuperado de https://www. sciencedirect.com/science/article/pii/S0264275111001612 
Vargas Llosa, M. (2012). La civilización del espectáculo. Madrid, España: Alfaguara.

Villegas, G. (2016). Frenan demolición de casona en Monterrey. Refor$m a, 18$ de noviembre, p. 1. Recuperado de https://www.reforma.com/ aplicacioneslibre/preacceso/articulo/default.aspx?id=987684\&urlre direct=https://www.reforma.com/aplicaciones/articulo/default.aspx ?id $=987684$

Villarreal González, A., Gasca Sánchez, F. M. y Flores Segovia, M. A. (2016). Patrones de aglomeración espacial de la industria creativa en el Área Metropolitana de Monterrey. Estudios Demográficos y Urbanos, 31(2), 331-383. Recuperado de https://estudiosdemograficosyurbanos. colmex.mx/index.php/edu/article/view/1591

Yúdice, G. (2008). Modelos de desarrollo cultural urbano: ¿gentrificación o urbanismo social? Alteridades, 18(36), 47-61. Recuperado de https:// www.redalyc.org/articulo.oa?id=74716004005

Zaid, G. (2013). Dinero para la cultura. Ciudad de Mexico: Debate.

Zukin, S. (1982). Loft living: Culture and capital in urban change. Baltimore, MD: Johns Hopkins University Press.

Zukin, S. (1987). Gentrification: Culture and capital in the urban core. Annual Review of Sociology, 13(1), 129-147. Recuperado de https://www. annualreviews.org/doi/abs/10.1146/annurev.so.13.080187.001021

Zukin, S. (1995). The culture of cities. Oxford / Cambridge, Reino Unido: Blackwell Publishers.

\section{Acerca de los autores}

Fabiola Garza-Rodríguez es doctora y maestra en Sostenibilidad por la Universitat Politècnica de Catalunya, España, y arquitecta por la Universidad Autónoma de Nuevo León (UANL), México. Actualmente es profesora en la Facultad de Arquitectura de la UANL, donde imparte clases tanto a nivel licenciatura como a nivel posgrado. Sus líneas de investigación se centran en: impactos sociourbanos de la cultura, gobernanza, gestión y participación ciudadana, y paisajismo e infraestructura verde en entornos urbanos. ORCID: http://orcid.org/0000-0001-5971-0478

Elisabet Roca es doctora en Ciencias Ambientales por la Universitat Autònoma de Barcelona, España. Actualmente es profesora e investigadora en el Instituto de Ciencia y Tecnología de la Sostenibilidad en la Universitat Politècnica de Catalunya, España, donde, desde el Laboratorio de Estu- 
dios Sociales de la Ingeniería Civil, desarrolla sus líneas de investigación en: gobernanza y gestión de zonas costeras, impacto socioambiental de las intervenciones urbanas, soluciones basadas en la naturaleza y servicios ecosistémicos urbanos, inclusión social y participación en políticas de movilidad. ORCID: https://orcid.org/0000-0001-9432-0029

Míriam Villares es Catedrática de Escuela Universitaria en la Universitat Politècnica de Catalunya, España (UPC). Es docente de Urbanismo en Ingeniería Civil y Ciencias Sociales en el Máster Universitario en Ciencia y Tecnología de la Sostenibilidad. Es investigadora del Laboratorio de Estudios Sociales de la Ingeniería Civil (LESEC-UPC). Ha participado en proyectos de investigación europeos y ha coordinado proyectos financiados por el Ministerio de Economía, Industria y Competitividad de España. Actualmente es secretaria del Instituto Universitario de Investigación en Ciencia y Tecnologías de la Sostenibilidad (ISST-UPC). ORCID: https://orcid.org/00000001-6910-931X

Recepción: 14 de junio de 2018. Aceptación: 25 de enero de 2019. 
